Journal of

Applied

Crystallography

ISSN 1600-5767

\title{
Texture analysis with a time-of-flight neutron strain scanner
}

\section{Florencia Malamud, Javier R. Santisteban, Miguel Angel Vicente Alvarez, Raúl Bolmaro, Joe Kelleher, Saurabh Kabra and Winfried Kockelmann}

J. Appl. Cryst. (2014). 47, 1337-1354

Copyright (C) International Union of Crystallography

Author(s) of this paper may load this reprint on their own web site or institutional repository provided that this cover page is retained. Republication of this article or its storage in electronic databases other than as specified above is not permitted without prior permission in writing from the IUCr.

For further information see http://journals.iucr.org/services/authorrights.html

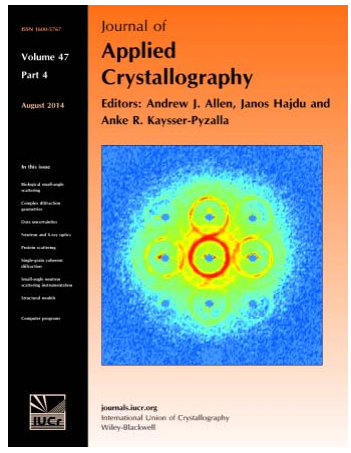

Many research topics in condensed matter research, materials science and the life sciences make use of crystallographic methods to study crystalline and non-crystalline matter with neutrons, X-rays and electrons. Articles published in the Journal of Applied Crystallography focus on these methods and their use in identifying structural and diffusioncontrolled phase transformations, structure-property relationships, structural changes of defects, interfaces and surfaces, etc. Developments of instrumentation and crystallographic apparatus, theory and interpretation, numerical analysis and other related subjects are also covered. The journal is the primary place where crystallographic computer program information is published.

Crystallography Journals Online is available from journals.iucr.org 
Journal of

Applied

Crystallography

ISSN 1600-5767

Received 27 March 2014

Accepted 31 May 2014

\title{
Texture analysis with a time-of-flight neutron strain scanner
}

\author{
Florencia Malamud, ${ }^{\mathrm{a} *}$ Javier R. Santisteban, ${ }^{\mathrm{a}}$ Miguel Angel Vicente Alvarez, ${ }^{\mathrm{a}}$ Raúl \\ Bolmaro, ${ }^{\text {b }}$ Joe Kelleher, ${ }^{c}$ Saurabh Kabra ${ }^{c}$ and Winfried Kockelmann ${ }^{c}$
}

\begin{abstract}
aLaboratorio de Física de Neutrones, Centro Atómico Bariloche, Avenida Bustillo 9500, San Carlos de Bariloche, Rio Negro 8400, Argentina, 'b Instituto de Física Rosario, CONICET-UNR, 27 de Febrero 210bis, Rosario, Santa Fe 2000, Argentina, and ${ }^{\mathbf{c}}$ Rutherford Appleton Laboratory, ISIS Neutron and Muon Source, Harwell Oxford, Didcot OX11 OQX, UK. Correspondence e-mail: fmalamud@cab.cnea.gov.ar
\end{abstract}

\begin{abstract}
A time-of-flight (TOF) neutron strain scanner is a white-beam instrument optimized to measure diffractograms at precise locations within bulky specimens, typically along two perpendicular sample orientations. Here, a method is proposed that exploits the spatial resolution $(\sim 1 \mathrm{~mm})$ provided by such an instrument to determine in a nondestructive manner the crystallographic texture at selected locations within a macroscopic object. The method is based on defining the orientation distribution function (ODF) of the crystallites from several incomplete pole figures, and it has been implemented on ENGIN-X, a neutron strain scanner at the ISIS facility in the UK. This method has been applied to determine the texture at different locations of $\mathrm{Al}$ alloy plates welded along the rolling direction and to study a $\mathrm{Zr} 2.5 \% \mathrm{Nb}$ pressure tube produced for a CANDU nuclear power plant. For benchmarking, the results obtained with this instrument for samples of ferritic steel, copper, Al alloys and $\mathrm{Zr}$ alloys have been compared with measurements performed using conventional X-ray diffractometers and more established neutron techniques. For cases where pole figure coverage is incomplete, the use of TOF neutron transmission measurements simultaneously performed on the specimens is proposed as a simple and powerful test to validate the resulting ODF.
\end{abstract}

Spatially resolved texture analysis would also be useful for the characterization of modern manufacturing methods, as well as in the quantification of the resulting residual stress distributions. For instance, welding processes introduce profound changes in microstructure, locally affecting grain size, composition and mechanical properties of the material. Residual stress analyses by neutron diffraction do consider the effect of local compositional changes through measurement of unstressed references taken far from the weld pool and heataffected zones, but changes in elastic constants (mainly due to texture) are usually dismissed, because of the experimental complexity involved in such measurements (Fitzpatrick \& Lodini, 2003). This has a direct impact on the accuracy of residual stress measurement within the weld pool of stainless steel welds and other face-centred cubic materials (Bouchard et al., 2005).

In principle, spatially resolved texture measurements could be performed on neutron strain scanners, which allow diffraction measurements to be done deep within engineering components and structures (Allen et al., 1985; Hutchings et al., 2004). These instruments are neutron diffractometers optimized for precise and fast determination of the positions of diffraction peaks obtained from a small region of a bulk object 
(the gauge volume), along with easy alignment of the momentum exchange vector to a direction of interest in the specimen. The elastic strain within this volume along the specified direction can be found from comparison of the measured peak position with the position measured for a stress-free reference. In practice, determination of the full strain tensor requires measurements along several directions of the object, which demands rotation and/or tilting of the specimen in the diffractometer. Hence, texture analysis within a bulky object could be effectively performed in a neutron strain scanner that offers a spatial resolution of $\sim 1 \mathrm{~mm}$. This has been effectively implemented in StressSpec, a constantwavelength neutron diffractometer at the FRM-2 reactor, Germany (Brokmeier et al., 2011), where three monochromators are available to optimize experimental arrangements for either strain or texture determinations. Successful implementation of the technique requires proper handling of the attenuation occurring within the specimen and, for practical reasons, determination of the orientation distribution function (ODF) of crystallites from a limited number of incomplete pole figures.

Time-of-flight (TOF) neutron diffraction uses a polychromatic neutron beam, with the advantage that several crystalline reflections can be measured simultaneously for a given specimen direction, allowing the determination of several (incomplete) pole figures from a single experimental arrangement. Hence, fast texture measurements are routinely performed on instruments such as HIPPO (Wenk et al., 2003) or GEM (Kockelmann, Chapon \& Radaelli, 2006), which are furnished with many detectors covering large fractions of the complete pole figure. However, the spatial resolution of these instruments is limited to $\sim 1 \mathrm{~cm}$. On the other hand, TOF neutron strain scanners [such as ENGIN-X (Santisteban et al., 2006), Vulcan (Wang et al., 2006), TAKUMI (Suzuki et al., 2013), SMARTS (Bourke et al., 2002) or Epsilon (Walther et al., 2000)] have a typical spatial resolution of $\sim 1 \mathrm{~mm}$ but a limited angular coverage, with two detector banks centred at scattering angles of $\pm 90^{\circ}$. Hence, many sample orientations together with long counting times are in principle necessary for quantitative texture analysis in TOF neutron strain scanners. Here we present a method that has been implemented on the ENGIN-X strain scanner, for texture analysis with a spatial resolution of $\sim 1 \mathrm{~mm}$. The method is based on the determination of several incomplete pole figures after splitting of the detector arrays into several units of smaller angular coverage. Determination of the orientation distribution function of crystallites from the incomplete pole figures is accomplished by means of MTEX (Hielscher \& Schaeben, 2008), a texture analysis library based on an algorithm especially suited for sharp textures and high-resolution pole figures measured on scattered specimen directions. Owing to high demand, available experimental time is normally scarce in neutron strain scanning experiments, so the method aims to define a reasonable ODF from a reduced number of sample orientations. In such cases, a confirmation of the accuracy of the resulting ODF is accomplished by comparison with simultaneous measurements of the TOF transmitted neutron spectra, whose magnitude is greatly affected by the texture of the material. In $\$ 2$ we present the experimental arrangement, the basic equations of the proposed technique and the actual implementation of the method in NyRTex, a freely available MATLAB (The MathWorks Inc., Natick, MA, USA) package. The accuracy of the proposed data analysis strategy is demonstrated in $\S 3$ through test experiments on ferritic steel, copper and aluminium specimens that have also been characterized by other diffraction techniques. Spatially resolved experiments are demonstrated on Al2024 welds and on $\mathrm{Zr} 2.5 \% \mathrm{Nb}$ pressure tubes obtained with a limited coverage of the pole figures. The practical application and resulting uncertainty of the proposed method are discussed in $\$ 4$.

\section{Texture-analysis methodology}

\subsection{ENGIN-X description}

ENGIN-X is a TOF neutron strain scanner, i.e. a diffractometer optimized to measure the precise position of diffraction peaks from a small well defined volume of a bulk component. Detailed information about the instrument is given by Santisteban et al. (2006), so only a brief description is provided here. A beam of neutrons from a pulsed source and a liquid methane moderator travels along a curved neutron guide and impacts on a sample at $\sim 50 \mathrm{~m}$ from the source. Fig. 1(a) shows a schematic aerial view of ENGIN-X at the sample position, which is representative of other TOF neutron strain scanners. The collimated beam of polychromatic neutrons impacts on a bulky sample, here represented by a tube. The neutrons scattered in two directions perpendicular to the incident beam are detected by detection banks located at $\sim 1.5 \mathrm{~m}$ from the sample position and centred at scattering angles of $\pm 90^{\circ}$ (north and south banks in the figure), covering $\pm 16^{\circ}$ in the horizontal plane and $\pm 21^{\circ}$ in the vertical plane. Each detector bank is composed of 1200 individual detectors arranged in 240 columns and five rows, each detector being a $196 \mathrm{~mm}$ high $\times 3 \mathrm{~mm}$ wide $\mathrm{ZnS} /{ }^{6} \mathrm{Li}$ scintillator. A single detection bank covers a total detector area of $1.4 \mathrm{~m}^{2}$, which represents about $5 \%$ of the total $4 \pi$ solid angle. The two $\sim 90^{\circ}$ scattering angles with such small angular coverage allow the determination of the elastic strain tensor along two mutually perpendicular directions of the object being investigated. The cross section and divergence of the incident beam are defined by three pairs of adjustable slits (only one is shown in the figure). The region of the object that is gauged by the instrument corresponds to a cuboid, which results from the intersection of the incident and diffracted beams (represented by a small dark rectangle in the figure). The divergence of the scattered beam $(\sim 0.002$ radians $)$ and the centre of this gauge volume are defined by radial collimators. The size of the gauge volume is changed by setting different slit apertures and choosing among five different collimator sizes (providing dimensions of $0.5,1,2,3$ and $4 \mathrm{~mm}$ ). In order to investigate different specimen directions, the orientation of the sample can be controlled by a choice of different goniometers, depending on the size and weight of the specimen being 
investigated. In standard strain scanning experiments, all neutrons arriving at a detection bank are combined into a single diffractogram by a post-analysis routine. A typical diffractogram for $\mathrm{Zr} 2.5 \% \mathrm{Nb}$ material from a pressure tube used in CANDU power plants is shown in Fig. 2(a). As observed in the figure, the use of a polychromatic beam provides information about multiple crystal families (and hence crystal orientations) at a fixed scattering angle. The diffractogram in Fig. 2( $a$ ) has been recorded by the north bank with the sample oriented as shown in Fig. 1(a). Hence, each diffraction peak ( $k h i l)$ provides information about the number of crystallites having their (khil) plane normals centred around the radial direction of the tube. On the other hand, the south bank gives information about those crystallites with their (khil) plane normals centred around the tube hoop direction. The directions and solid angles covered by each bank in Fig. 1(a) have been identified in the pole figure represented in Fig. 1(b). Determination of a complete pole
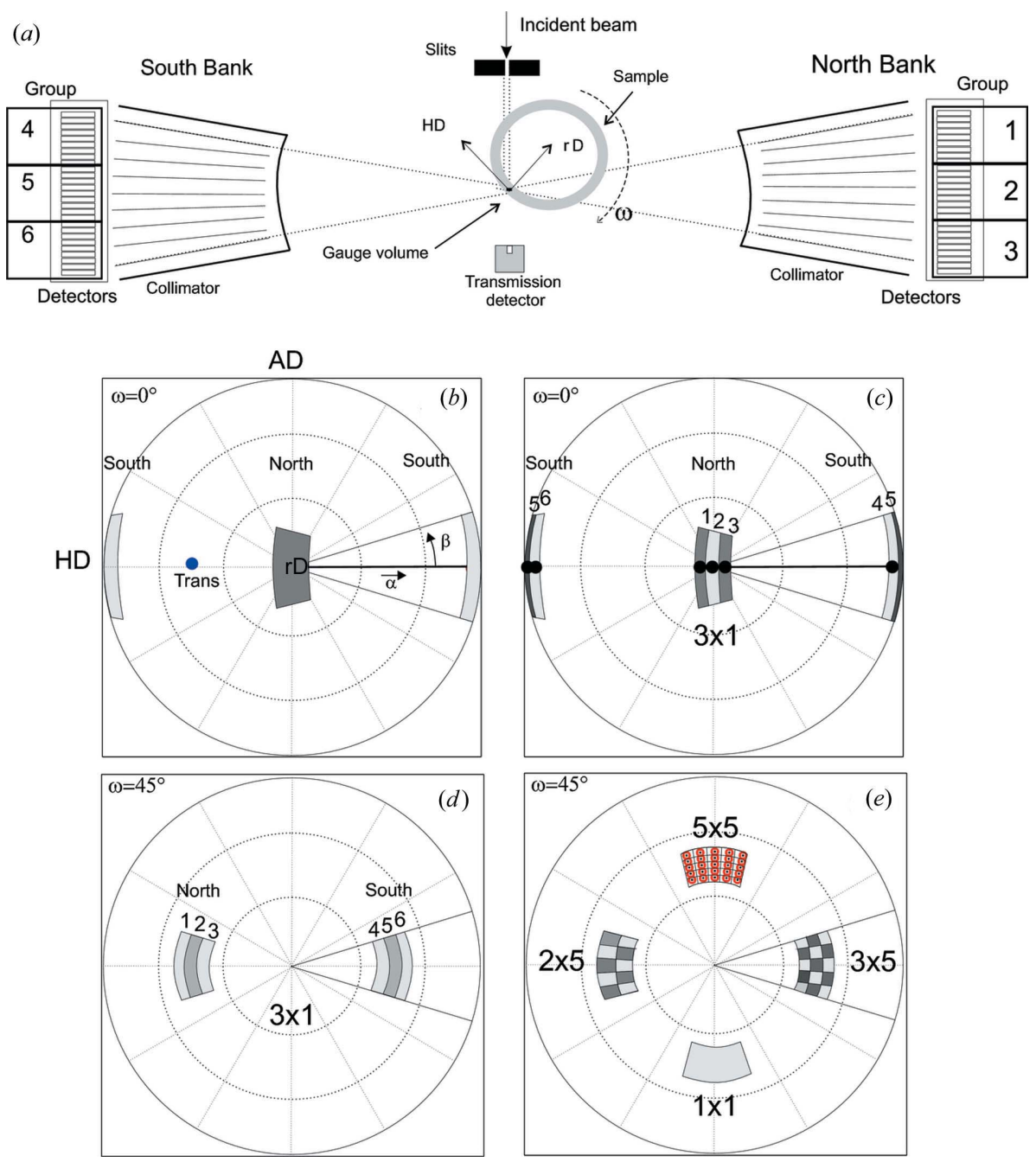

Figure 1

(a) Schematic diagram of the ENGIN-X time-of-flight neutron strain scanner, with a tube at the sample position. $(b)-(e)$ Pole figure coverage corresponding to different gridding schemes of the detection banks and for different sample orientations. The $3 \times 1$ (horizontal $\times$ vertical) gridding scheme shown in $(a)$ is represented in $(c)$ and $(d)$ for two orientations of the specimen. HD hoop direction, $\mathrm{rD}$ radial direction, $\mathrm{AD}$ axial direction. figure would require several rotations and tilts of the sample. As two specimen directions are measured simultaneously by the instrument, full coverage of the orientation space using a typical $5 \times 5^{\circ}$ grid would require $(72 \times 18) / 2=648$ orientations, a prohibitively large number when considering the measurement time and very high demand for such instruments. Moreover, such information would be certainly redundant for defining the ODF of typical metal products, as $\sim 15$ complete pole figures are obtained from the experiment. This calls for a different strategy in order to make texture measurement practical and feasible in common applications.

\subsection{Sub-banks and angular resolution}

Each diffraction bank covers $\pm 16^{\circ}$ in the horizontal plane and $\pm 21^{\circ}$ in the vertical plane. For the $\sim 90^{\circ}$ scattering angle, this results in a pole figure coverage of $\pm 8-16^{\circ}$ in the diffraction plane and $\pm 21-42^{\circ}$ out of this plane. This relatively large solid angle, represented in Fig. 1(b), can be sub-divided into smaller detection units in order to improve the angular resolution and increase the number of directions explored for a fixed orientation of the specimen. This is done by histogramming the 1200 detection elements composing a bank into smaller groups, with each of these virtual detectors hence covering a smaller solid angle. As an example, in Fig. 1(a) the two detection banks have been sub-divided into three smaller units in the diffraction plane, and each sub-unit has been identified by a number (1-6). For this case, each virtual detector is composed of a group of $80 \times 5=400$ detectors (horizontal $\times$ vertical), covering $\sim 42^{\circ}$ in the vertical plane and $\sim 5^{\circ}$ in the horizontal plane. The pole figure in Fig. 1(c) indicates the locations and approximate solid angles covered by those virtual detectors using this $3 \times 1$ gridding scheme (horizontal $\times$ vertical). As mentioned above, several rotations of the specimen are required in order to achieve reasonable coverage of the pole figure, by moving the virtual detectors into different locations of the pole figure. Fig. 1(d) shows the effect of a $45^{\circ}$ rotation around a vertical axis (i.e. normal to the page) on the location of the virtual detectors within the pole figure. More efficient virtual detector arrangements can be defined, depending on the specific problem and available counting time, always within the 
constraint imposed by the $240 \times 5$ geometric array of physical detectors. At present, this fixes a value of $42^{\circ} / 5 \simeq 8^{\circ}$ for the minimum angular coverage achievable in the vertical plane. Fig. 1(e) shows the approximate angular coverage of the individual virtual detectors for four possible virtual detector configurations with the ENGIN-X instrument.

\subsection{Direct and inverse pole figures}

Construction of an experimental pole figure $P_{h k l}(\alpha, \beta)$ requires a quantitative determination of the integrated peak area recorded by a virtual detector along a certain direction of the specimen ( $\alpha=$ latitude, $\beta=$ longitude; Fig. $1 b$ ), as compared to the value registered by the same detector for an ideal, perfectly random polycrystalline sample having the same microstructure as the material under study. This is seldom possible, so in practice several corrections need to be performed to the peak area measured by each individual virtual detector, in order to produce a combined pole figure from data registered by different virtual detectors. In addition to the (unknown) texture factor, the integrated intensity of a diffraction peak measured in a TOF diffractometer depends on the scattering power $\left(G_{h k l}\right)$ and linear absorption coefficient $\left(\mu_{h k l}\right)$ of the sample, as well as on an instrumental factor $\left(\Phi_{\text {inst }}^{j}\right)$ specific to each virtual detector $(j)$ (Santisteban et al., 2006). Hence, in this work the pole figure intensity $\left(P_{h k l}\right)$ recorded by a virtual detector is obtained from the measured integrated peak area $\left(I_{h k l}\right)$ according to

(a)

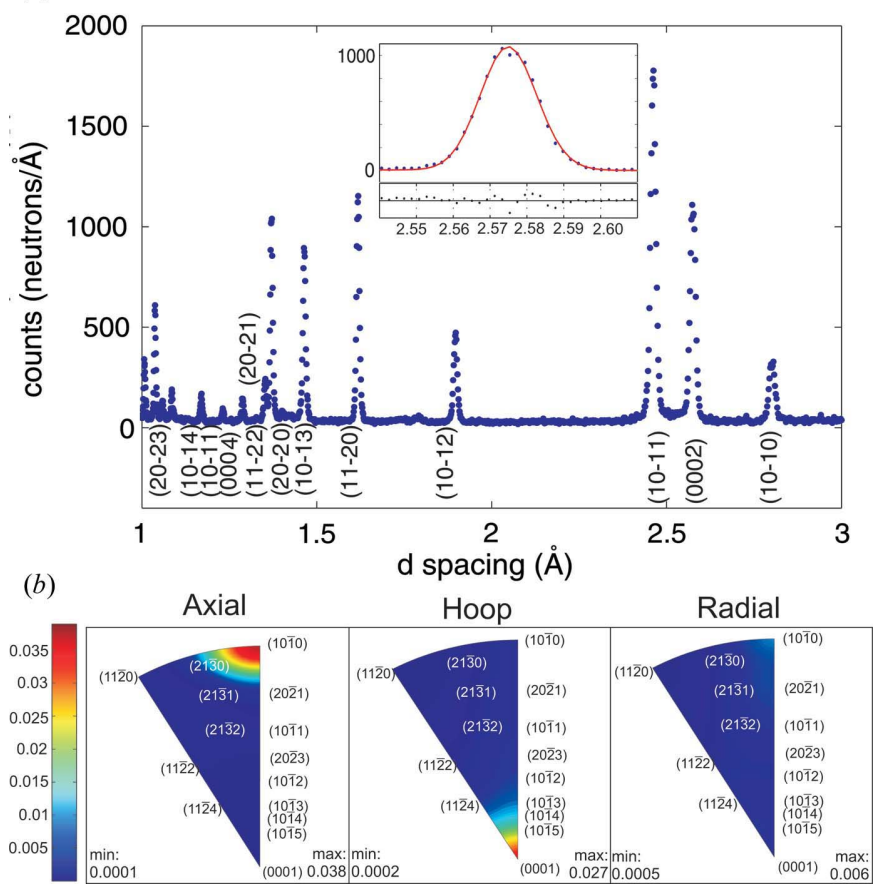

Figure 2

(a) Typical time-of-flight diffractogram for a $\mathrm{Zr} 2.5 \% \mathrm{Nb}$ pressure tube measured on ENGIN-X. The data were recorded by detector number 2 in the experimental arrangement shown Fig. 1(a). (b) Inverse pole figures for the three principal axes of the tube, each one produced from data registered by a single virtual detector.

$$
P_{h k l}(\alpha, \beta)=I_{h k l}(\alpha, \beta) /\left[G_{h k l} \exp \left(-l \mu_{h k l}\right) \Phi_{\text {inst }}^{j}\right],
$$

with $l$ the average length of the neutron path inside the specimen. The scattering power is given by

$$
G_{h k l}=m_{h k l}\left|F_{h k l}^{2}\right| d_{h k l}^{4} / v_{0}^{2},
$$

where $v_{0}$ is the volume per atom, $F_{h k l}^{2}$ the structure factor (including the Debye-Waller factor), $m_{h k l}$ the multiplicity of the reflection and $d_{h k l}$ the interplanar distance of the particular reflection. The instrumental factor is given by

$$
\Phi_{\text {inst }}^{j}=\Phi_{0}^{j}(\lambda) \varepsilon_{\mathrm{j}}(\lambda) \frac{h_{\mathrm{j}}}{\pi L_{j}} \alpha_{\mathrm{in}}^{\mathrm{vert}} \sin ^{2} \theta_{\mathrm{B}, j}\left[\left(\alpha_{\mathrm{in}}^{\mathrm{hor}}\right)^{2}+\left(\alpha_{j}^{\mathrm{hor}}\right)^{2}\right]^{1 / 2} \delta V,
$$

where $\theta_{\mathrm{B}, j}, L_{j}$ and $h_{j}$ are, respectively, the mean Bragg angle, the distance to the sample and the height of the $j$ th virtual detector; $\Phi_{0}^{j}(\lambda)$ and $\varepsilon_{j}(\lambda)$ are, respectively, the incident neutron flux and detection efficiency at the wavelength $\lambda=$ $2 d_{h k l} \sin \theta_{\mathrm{B}, j} ; \delta V$ is the gauge volume, i.e. the volume of the sample illuminated by the neutron beam that is effectively seen by the detector (as illustrated in Fig. $1 a$ ); $\alpha_{\text {in }}^{\text {hor }}$ and $\alpha_{\text {in }}^{\text {vert }}$ are the divergences of the incident beam in the diffraction plane and normal to it, respectively; and $\alpha_{j}^{\text {hor }}$ is the divergence of the scattered beam seen by the detector, as allowed by the radial collimator. The wavelength dependence of $\Phi_{\text {inst }}^{j}$ is contained in the product term $\Phi_{0}^{j}(\lambda) \varepsilon_{j}(\lambda)$, usually approximated by measuring the spectra scattered by a $\mathrm{V} 3 \% \mathrm{Nb}$ sample under the same experimental conditions, which is assumed to be a perfectly elastic incoherent scatterer.

Using equation (1), inverse pole figures can be directly constructed from the data registered by a single virtual detector. As an example, Fig. 2(b) shows inverse pole figures measured for the three principal axes for the $\mathrm{Zr} 2.5 \% \mathrm{Nb}$ pressure tube depicted in Fig. 1(a). The inverse pole figure for the radial direction has been directly obtained from the diffractogram shown in Fig. 2(a), which corresponds to the data recorded by detector number 2 in the experimental arrangement shown Fig. 1(a). The inverse pole figure for the hoop direction was produced from the diffractogram registered by detector number 5 , whilst that for the axial direction was obtained after a $90^{\circ}$ rotation of the specimen around a horizontal axis. Important integral parameters of hexagonal materials such as Kearns factors can be approximated from such inverse pole figures (Kearns, 1965). Direct pole figures for specific $h k l$ reflections are produced by plotting the $P_{h k l}$ obtained by all virtual detectors and specimen orientations explored by the experiment into a single figure. Examples of such (incomplete) experimental pole figures are found in the insets of Figs. 3-7 below. Details about the samples, instrument configurations, counting times and computational methods used to produce such pole figures are provided later in the text.

As mentioned above, determination of complete pole figures would require rather long counting times, which are unlikely to be available in normal use of neutron strain scanners. Hence, in practice we aim to achieve a reasonable description of the ODF from measurements performed on a 
limited number of specimen orientations. This goal is not unreasonable, because many reflections are measured simultaneously in TOF diffraction. However, some important features of the ODF could be missing in such an approach, because of incomplete coverage of the pole figure. In order to avoid or mitigate such problems, we propose to register during the experiment the neutron signal transmitted by the sample, as a function of TOF. As shown in the next section, the wavelength-dependent transmission is highly sensitive to the ODF of the material, so it can provide an additional criterion to assess the soundness of the resulting texture analysis.

\subsection{Transmitted signal and total cross section calculation}

Determination of the transmitted neutron spectrum is very simple. It only requires a single TOF detector aligned with the neutron beam, as shown schematically in Fig. 1(a). Owing to the high intensity available in TOF neutron strain scanners, measurement times are relatively short, typically of the order of $1-5 \mathrm{~min}$. The transmission of a specimen as a function of neutron wavelength is obtained by comparing the spectrum registered with the sample placed in the neutron beam $(\Phi)$ with the spectrum registered for the direct beam $\left(\Phi_{0}\right)$,

$$
\operatorname{Tr}(\lambda, \tau)=\Phi(\lambda, \tau) / \Phi_{0}(\lambda)
$$

where $\tau$ indicates the direction of the transmitted neutron beam in the coordinate system of the sample, assuming normalization of the incident neutron flux for sample-in and direct-beam measurements and neglecting background contributions for sample-in and sample-out measurements. In a first approximation, this transmission is directly related to the microscopic total cross section $\left(\sigma_{\text {tot }}\right)$ of the specimen by

$$
\operatorname{Tr}(\lambda, \tau)=\exp \left[-n l \sigma_{\text {tot }}(\lambda, \tau)\right]
$$

where $l$ is the length of the neutron path within the sample in the direction of the neutron beam and for polycrystalline materials $n$ is the number of unit cells per unit volume. The total cross section includes all processes that remove neutrons from the incident beam, i.e. Bragg reflection, diffuse scattering and absorption. The two latter contributions have smooth dependences on neutron wavelength, and methods for their calculation are found in several textbooks (Marshall \& Lovesey, 1971, etc.). By contrast, the contribution to the total cross section due to Bragg reflection on the crystal planes presents sharp changes in wavelength, as shown in Fig. 3(a) for a sheet of ferritic steel. The dotted green line is the total cross section expected for a powder sample, whilst the blue crosses are experimental values measured for a direction intermediate between the normal and transverse directions of the sheet, identified by a black ' $x$ ' in the pole figure shown in the inset. The sharp steps observed in the plot are the so-called Bragg edges and occur because of reflection on the crystal planes. For a crystal family $\{h k l\}$, the Bragg angle increases as the wavelength increases, $\lambda=2 d_{h k l} \sin \theta_{\mathrm{B}}$, until $2 \theta_{\mathrm{B}}$ is equal to $180^{\circ}$. At wavelengths greater than $2 d_{h k l}$ no Bragg reflection on this particular $\{h k l\}$ family can occur, resulting in a drop in the total cross section, i.e. a sharp increase in the transmitted intensity. The height of the $(h k l)$ Bragg edge gives a direct measure of the number of crystallites having their $\{h k l\}$ planes normal to the incoming beam. The height of Bragg edges changes drastically for textured materials and can even be zero along specific directions of a specimen, as observed in the experimental data of Fig. 3(a) for the 200 reflection. For most metallic materials this coherent scattering component represents the largest contribution to the total cross section for the wavelengths of thermal neutrons. The differences between the experimental results (blue crosses) and the theoretical curve evaluated for a powdered specimen (dotted green line) reveal that the total cross section is highly sensitive to the crystallographic texture of the material. The total cross section

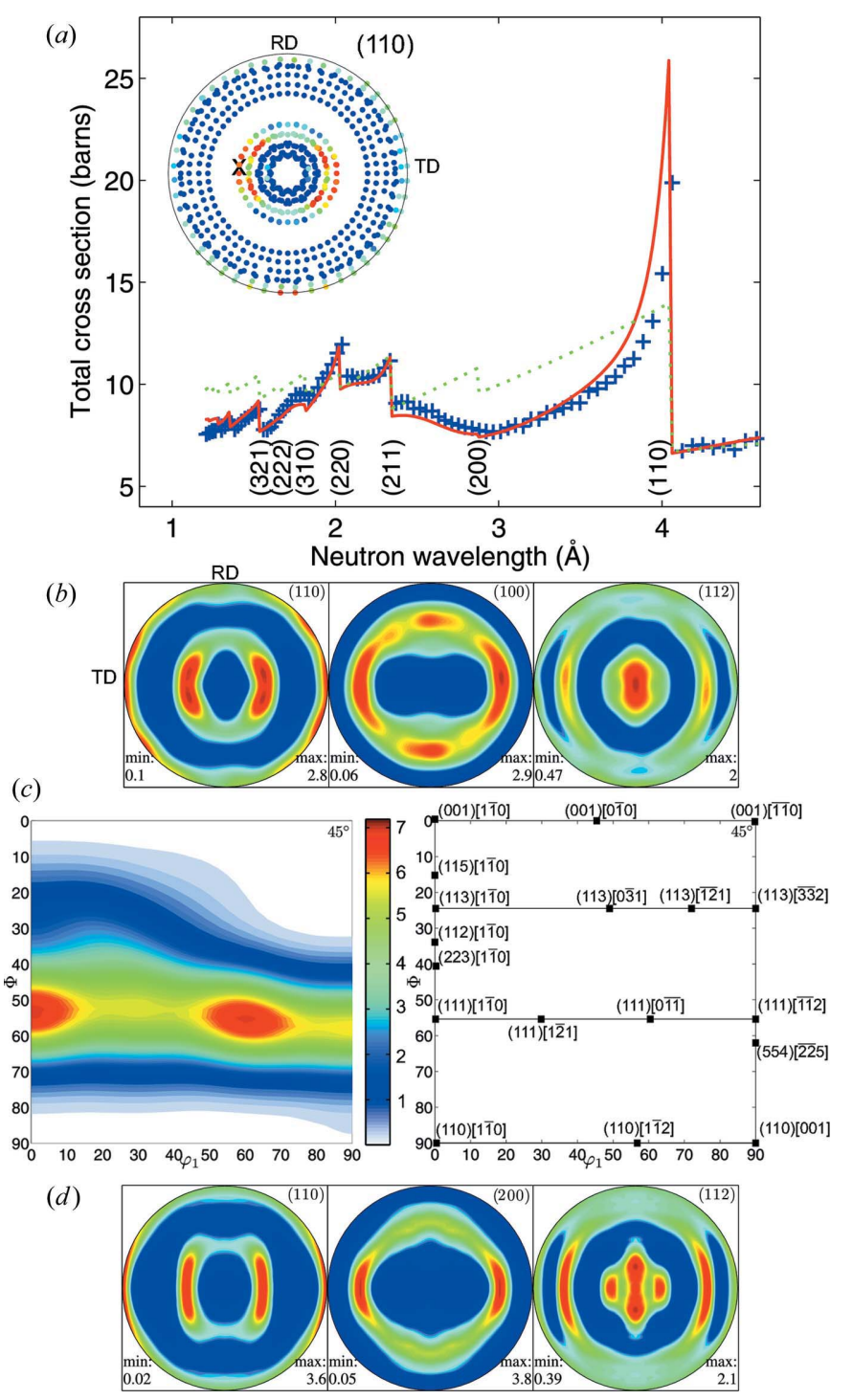

Figure 3

(a) Experimental (blue crosses) and calculated (red line) neutron total cross section of a low-carbon steel sheet along the direction indicated as ' $X$ ' in the experimental pole figure shown in the inset. $(b)$ Recalculated pole figures from the ODF calculated with MTEX from experimental pole figures such as that of the inset. (c) Cut of the ODF at $\varphi_{2}=45^{\circ}$, showing the main texture components of the material. (d) Recalculated pole figures from accompanying X-ray diffraction experiments. RD rolling direction, TD transverse direction, ND normal direction. 
component due to Bragg reflection $\left(\sigma_{\text {coh }}^{\mathrm{el}}\right)$ along a specimen direction $\tau$ can be directly calculated from the pole figures of the material (Santisteban et al., 2012):

$$
\sigma_{\mathrm{coh}}^{\mathrm{el}}(\tau, \lambda)=\frac{\lambda^{2}}{4 v_{0}} \sum_{h k l}^{2 d_{h k l}<\lambda}\left|F_{h k l}\right|^{2} d_{h k l} R\left(\tau, \lambda, d_{h k l}\right) .
$$

The factor $R$ is given by a line integral around a circle in the $P_{h k l}^{\tau}(\alpha, \beta)$ pole figure, where the superscript $\tau$ indicates that the pole figure has been rotated in order to have the direction $\tau$ at its centre:

$$
R\left(\tau, \lambda, d_{h k l}\right)=\int_{0}^{2 \pi} P_{h k l}^{\tau}\left[\frac{\pi}{2}-\arcsin \left(\frac{\lambda}{2 d_{h k l}}\right), \beta\right] \mathrm{d} \beta .
$$

This expression essentially counts the number of crystallites whose $\tau$ directions make an angle $\alpha_{h k l}=(\pi / 2)-\arcsin [\lambda /$ $\left.\left(2 d_{h k l}\right)\right]$ with the incident neutron beam. The red solid line in Fig. 3(a) shows such a calculation for the ferritic steel, using the pole figures displayed in Fig. 3(b). For an isotropic specimen $R\left(\tau, \lambda, d_{h k l}\right)=1$, and we recover the expression for the elastic coherent total cross section given by Fermi \& Marshall (1947), which corresponds to the dotted green curve in Fig. 3(a). Comparison between calculated and experimental total cross sections is a thorough check of the resulting texture analysis. Hence, the total cross section measured along several directions of the specimen can assess the quality of texture analyses based on incomplete pole figures. Good agreement between experiment and calculation means that the texture model has effectively captured all main features of the ODF. This is because sharp features of the ODF are revealed plainly on the wavelength scale, as inferred from equation (7) and shown by Santisteban (2005) for mosaic crystals. Hence, we propose measuring the total cross section of the specimen along a limited number of directions, to complement the texture analysis based on incomplete pole figures from a number of detector groups. For this purpose, here we have used a transmission detector available in ENGIN-X. The detector is pixelated, composed of a $10 \times 10$ array of $2 \mathrm{~mm}$ cubes of scintillating glass GS20 (Santisteban et al., 2002), but such spatial resolution has not been exploited in the present work.

It is important to keep in mind that the measured transmission is along the full path of the beam through the sample, which might differ significantly from the gauge volume under investigation in diffraction (and from which the pole figures are derived). This limits the usefulness of this controlling approach somewhat, particularly in cases in which texture from small gauge volumes is of specific interest. It must also be noted that depending on the symmetry of the specimen, only a limited number of directions could actually be measured in transmission. For instance, a proper total cross section measurement would not be feasible for the tube orientation represented in Fig. 1( $a)$, because in such a case the neutron beam is traversing the specimen at two locations that have different orientations in a cylindrical reference system.

\subsection{NyRTex}

All of the tasks involved in the proposed texture analysis procedure have been implemented within the MATLAB computing language through NyRTex, a freely available package specifically written for this purpose. NyRTex performs the following operations:

(i) Definition of sub-banks: the program splits the $42 \times 32^{\circ}$ solid angle covered by each ENGIN-X bank into smaller groups, as exemplified in Fig. 1. The definition of sub-groups is very versatile and results in a number of virtual detectors identified by azimuthal and polar scattering angles. After a measurement, a single diffractogram is produced for each virtual detector (like that shown in Fig. $2 a$ ), by adding the TOF spectra recorded by all individual detector elements belonging to the group into a common $d$-spacing scale. NyRTex makes extensive use of the Libisis library developed within MATLAB at the ISIS pulsed neutron source for analysing neutron scattering data (http://www.libisis.org).

(ii) Definition of peak areas: the integrated areas of selected peaks from the diffractograms are obtained by least-squares fitting to the experimental data, using a multi-peak fitting algorithm based on asymmetric peak profiles (convolution of a Gaussian with a truncated exponential; Kropff et al., 1982) on top of a linear background. A typical fit is shown in the inset of Fig. 2(a). For this particular case, the incident beam divergence has been deliberately increased, so the Gaussian contribution dominates the peak profile. NyRTex uses the MATLAB Optimization Toolbox (http://www.mathworks. com/products/optimization/) for an efficient least-squares minimization process.

(iii) Construction of experimental pole figures: the program uses the goniometer angles ascribed to each measurement run together with the peak areas obtained for all virtual detectors and corrects the intensities using equations (1)-(3), in order to produce direct (Fig. $3 b$ ) and inverse (Fig. $2 b$ ) pole figures.

(iv) Determination of the ODF: on the basis of the experimental pole figures and the symmetries of the crystal and the sample, the program performs a least-squares determination of the orientation distribution function of the crystallites using the MTEX algorithm (Hielscher \& Schaeben, 2008). This algorithm is especially well suited for sharp textures and high-resolution pole figures measured with respect to arbitrarily scattered specimen directions, e.g. by the ENGIN-X virtual detectors. The estimated ODF is computed as the solution of a minimization problem which is based on a model of the diffraction counts as a Poisson process.

(v) Calculations of derived properties: NyRTex calculates a number of physical properties from the refined ODF. Particularly relevant to the proposed method is the calculation of the elastic coherent cross section of the material based on equations (6) and (7). Another important property is the Kearns factor (Kearns, 1965), widely used in the characterization of hexagonal materials, calculated as

$$
f_{\tau}=\int_{0}^{2 \pi} \int_{0}^{\pi / 2} P_{000 l}^{\tau}(\alpha, \beta) \cos ^{2} \alpha \sin \alpha \mathrm{d} \alpha \mathrm{d} \beta .
$$


This factor is a quantification of the volume fractions of crystallites having basal poles aligned along a direction of interest in the sample, here denoted by $\tau$. As before, $P_{000 l}^{\tau}(\alpha, \beta)$ indicates that the pole figure has the direction $\tau$ at its centre. For three mutually orthogonal sample directions (e.g. hoop, radial, axial), the Kearns factors necessarily sum to one. This parameter is used to compute the effective value of a physical property along the (0001) crystal direction, projected into the specimen direction of interest; hence it includes the projection from all constituent crystallites. The Kearns factor is particularly useful as a quantitative assessment of the accuracy of the obtained pole figures.

For the tasks in steps (iii)-(v) and the plotting of recalculated pole figures, NyRTex uses the MATLAB MTEX Toolbox, which provides a powerful variety of object classes and methods specially designed for texture analysis (http:// code.google.com/p/mtex/).

\section{Experiments}

In order to assess and demonstrate the capability of the proposed method, we have performed experiments using the ENGIN-X instrument on a series of metallic samples made from several alloys presenting different crystallographic structures [body-centred cubic (b.c.c.), face-centred cubic (f.c.c.), hexagonal close packed (h.c.p.)]. The samples are representative of material problems where texture plays an important role, taken from archaeometry (copper alloys) as well as from the automotive (ferritic steel), aerospace (aluminium alloy) and nuclear (zirconium alloy) industries. The aim of this section is to illustrate the accuracy of the results achievable by the proposed method, in particular in relation to the necessary trade-off between counting time and pole figure coverage. In all examples shown below, we present one experimental pole figure that illustrates the incomplete pole figure coverage obtained during the experiment. For all cases except \$3.3.1, the ODFs obtained with the present method have been validated by complementary experiments performed on the same specimens using other, more conventional, techniques. This includes laboratory X-ray diffraction in reflection geometry, synchrotron X-ray diffraction in transmission geometry, constant-wavelength neutron diffraction and TOF neutron diffraction on instruments with detectors spanning large solid angles.

Besides those standard techniques, the resulting ODFs have also been validated through the method proposed here, i.e. measurements of the wavelength-dependent total cross section along specific sample directions. The transmission data presented here were measured on the ENGIN-X instrument at the same time as the texture experiments.

\subsection{Deep drawing steel}

One of the most important industrial uses of low-carbon steel sheets is in deep drawing applications. Drawability is the capacity of a material to achieve a high degree of plastic flow in the plane of a sheet, without significant reduction of the sheet thickness. As plastic flow is dominated by the creation and movement of dislocations gliding on given slip systems, drawability is highly dependent on crystallographic textures. In b.c.c. crystals, like ferritic steel, the most likely slip system is $\{110\}\langle 111\rangle$, and better drawability is achieved in materials having most of their grains with their $\{111\}$ planes on the sheet plane and a negligible fraction of crystals with $\{001\}$ planes on the sheet plane (Ray et al., 1993). Under this condition, most of the drawing deformation distributes on the sheet plane, with little reduction in thickness. Sheet manufacturing involves hot rolling, followed by cold rolling and annealing. Texture components develop during cold rolling, where thickness reductions of the order of $80 \%$ are applied, which are subsequently modified during annealing. Here, we have characterized the crystallographic texture of a low-carbon ferritic steel sheet of $0.8 \mathrm{~mm}$ thickness produced by Ternium Siderar, used for manufacturing car body panels. The composition of the material was $\mathrm{Fe}, 0.04 \mathrm{wt} \% \mathrm{C}, 0.17 \mathrm{wt} \% \mathrm{Mn}, 0.045 \mathrm{wt} \% \mathrm{Al}$, 0.03 wt $\%$ Si, 40 wt p.p.m. N. Ten discs of $10 \mathrm{~mm}$ diameter were machined out of the sheet and stacked together with Super Glue to produce a cylindrical specimen of $8 \mathrm{~mm}$ height. Neutron experiments were performed on ENGIN-X, using a gauge volume of $6 \times 4 \times 4 \mathrm{~mm}$ and an incident beam divergence, optimized for texture measurements, of $\sim 0.7 \times 0.8^{\circ}$ (horizontal $\times$ vertical). TOF diffractograms were collected at 31 specimen orientations using an Euler goniometer, with counting times of $\sim 5$ min per orientation. Data were analysed in a $2 \times 5$ (horizontal $\times$ vertical) gridding scheme, which gives to each virtual detector a coverage of $\sim 8 \times 8^{\circ}$ in the pole figure $\mathrm{SO}(2)^{\mathbf{1}}$ angular space (Fig. 1e). The experimental (110) pole figure in the inset of Fig. 3(a) shows the coverage achieved with this measurement strategy. The ODF of the material was calculated with MTEX from the (110), (200), (211), (220) and (310) experimental pole figures, considering triclinic sample symmetry. Fig. 3(b) shows recalculated pole figures and Fig. 3(c) a cut of the ODF at $\varphi_{2}=45^{\circ}$. The texture was also measured using a Panalytical X-pert MPD diffractometer, with $\mathrm{Cu} K \alpha$ radiation and an X-ray lens in the incident beam to guarantee a highly parallel and intense beam on a large part of the sample. Given the low penetration of the $\mathrm{Cu}$ radiation on $\mathrm{Fe}$ alloys, typically no more than $10 \mu \mathrm{m}$, the sample was carefully polished and electropolished to avoid surface damage that could influence surface texture. The analysis was performed by $W X p o p L A$, a current Windowsbased implementation of the popLA software (Kallend et al., 1991a,b). Defocusing correction and further treatment by harmonics and/or WIMV algorithms (Matthies, 2002) ensured the proper calculation of consistent ODFs and recalculated pole figures. These are shown in Fig. 3(d) and are found to be in very good agreement with those obtained on ENGIN-X. The most important structures found in the pole figures appear at the same angular positions and in almost all cases with similar intensity. Some minor differences are observed,

\footnotetext{
${ }^{1}$ The special orthogonal group, denoted $\mathrm{SO}(n)$, is the group of $n \times n$ orthogonal matrices with determinant 1 of a given dimension $n$, where the group operation is given by matrix multiplication. In dimensions 2 and 3, its elements are the usual rotation around a point $[\mathrm{SO}(2)]$ or a line $[\mathrm{SO}(3)]$.
} 
with neutron experiments predicting a more intense (200) pole at the top and bottom of the circular structure observed in this figure, and missing the two maxima at both sides of the elongated structure in the centre of the (112) pole figure. These discrepancies could be due to the lack of experimental observations close to the normal direction of the sheet (centre of the figures) and at $\alpha \simeq 45^{\circ}$, as indicated in the inset of Fig. 3(a).

In both texture determinations the low pole densities in the normal direction in the (200) pole figure indicate that the undesired $\{100\}$ texture component was effectively eliminated during annealing. A better insight on texture can be gained from the ODF cut, shown in Fig. 3(c), where the main texture components expected to appear in the material are well represented. The intense band found at $\Phi=55^{\circ}$ is the $\gamma$ fibre with $\{111\}$ planes parallel to the sheet, one of the components that results from cold rolling. The other (detrimental) component from cold rolling, with $\{001\}$ planes parallel to the sheet surface, has been eliminated by the choice of the correct heating rate during the final annealing. This has induced a recrystallization process that promotes the beneficial $\{111\}\langle 110\rangle$ component, corresponding to grains with a $\langle 110\rangle$ axis along the rolling direction. The presence of this intense fibre guarantees good drawability, yet the appearance of a major component, $\{111\}\langle 110\rangle$, dominating this band indicates that earing is likely to occur during drawing.

Fig. 3(a) shows the neutron total cross section measured for the specimen along a direction intermediate between the normal and transverse directions of the sheet, as indicated by a black ' $x$ ' in the experimental pole figure of the inset. The good agreement observed with the theoretical values (solid red line) evaluated using equations (6) and (7) from the ODF discussed above can provide confidence in the ODF analysis based on incomplete pole figures in cases where no contrast is available from other techniques.

\subsection{Replica sample as reference to ancient copper}

Texture analysis has been used to distinguish between possible manufacturing processes of ancient bronze objects (Siano et al., 2006; Kockelmann, Chapon \& Radaelli, 2006; Kockelmann, Chapon, Engels et al., 2006). For characterization of the manufacturing processes of prehistoric copper axes, Artioli (2007) performed an extensive program of crystallographic texture analysis on a large number of museum specimens on the GEM diffractometer, selected on the basis of their provenance and typological significance. To elucidate the process, Artioli also produced specimens in the laboratory under controlled conditions, in order to compare their textures with those from prehistoric times. In particular, an artificially prepared copper sample that shows a marked fibre texture due to columnar grain growth during crystallization has been used as a standard in comparisons of the performance of different instruments and data collection strategies. The specimen is an irregular parallelepiped of dimensions $\sim 20 \times 20 \times 10 \mathrm{~mm}$ and is shown in Fig. 4(b) fixed to the sample holder. We have measured this sample on ENGIN-X, using a gauge volume of
$6 \times 6 \times 4 \mathrm{~mm}$ and an incident beam divergence of $\sim 0.4 \times 0.5^{\circ}$ (horizontal $\times$ vertical). TOF diffractograms were collected at 57 specimen orientations using an Euler goniometer, with counting times of $\sim 7 \mathrm{~min}$ per orientation. Data were analysed in a $2 \times 5$ (horizontal $\times$ vertical) gridding scheme. The experimental (111) pole figure in the inset of Fig. 4(a) shows the coverage achieved with this measurement strategy. Points are not symmetrically distributed in the pole figure because the specimen orientations were optimized for a separate investigation, dealing with the dependence of the neutron transmission on sample orientation. The ODF of the material was calculated with MTEX from the (111), (100), (110) and (311) experimental pole figures considering triclinic sample symmetry. The recalculated pole figures shown in Fig. 4(c)

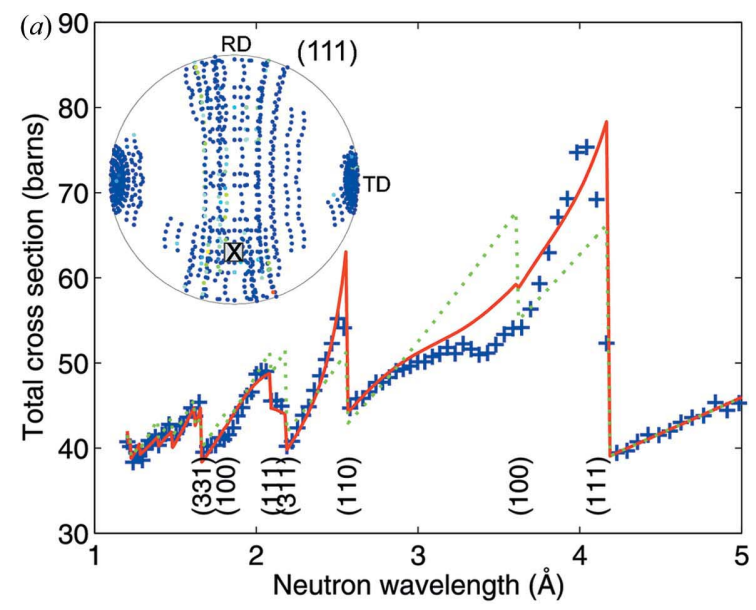

(b)
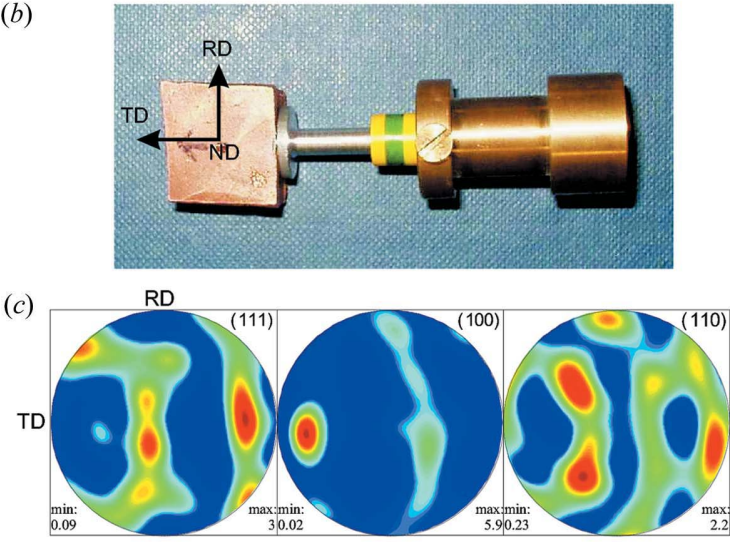

(d)

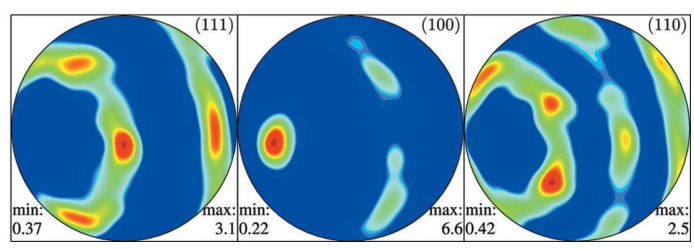

Figure 4

(a) Experimental (blue crosses) and calculated (red line) neutron total cross section of the copper specimen shown in $(b)$, produced to simulate ancient manufacturing techniques (Artioli, 2007). Measurements and calculations are for the direction indicated as ' $X$ ' in the experimental pole figure shown in the inset. $(c)$ and $(d)$ are recalculated pole figures, respectively, from the ODFs obtained from the present experiments and from additional experiments on the GEM neutron diffractometer (Kockelmann, Chapon, Engels et al., 2006). 
show good qualitative and quantitative agreement with results obtained on the same specimen using the GEM diffractometer (Fig. 4d) (Hannon, 2005). The $3.5 \pi$ solid angle of the GEM detectors was subdivided into 160 groups, and diffractograms were recorded for each of these groups for a single orientation of the specimen without reorientation. Texture analysis was performed using the MAUD program (Lutterotti et al., 1997), which uses a Rietveld-type fit of the 160 diffraction patterns and the WIMV method for determination of the ODF. Details about texture analysis using GEM are given by Kockelmann, Chapon \& Radaelli (2006). The pole figures reveal that crystallites are iso-oriented along one [100] direction in between the normal and transverse directions and approximately randomly distributed in other directions. The pole figures are the typical cube or rotated cube texture owing to recrystallization during thermal annealing of the rolled metal, with a uniaxial working force parallel to the normal direction. Its presence is taken as evidence of thermal treatment after mechanical working of a prehistoric object such as an axe, as the oriented recrystallization cannot take place without previous rotation of the crystallites due to plastic deformation (Artioli, 2007).

Fig. 4(a) shows the experimental total cross section measured along a direction intermediate between the normal direction and the rolling direction, together with the calculation based on the ODF determined on ENGIN-X. Good overall agreement is found between data and experiment, yet some differences are observed near the (100) Bragg edge, i.e. the reflection displaying the largest effects of preferred orientation.

\subsection{High-strength aluminium alloy plates}

High-strength $\mathrm{Al}$ alloys are widely used in the aerospace industry because of their high yield strength and good fatigue resistance. However, their industrial application remains somewhat limited owing to their highly anisotropic grain structures and properties. Texture control in aluminium alloys has been studied extensively for the improvement of formability (Engler \& Hirsch, 2002; Kong et al., 2010), because, as discussed for steel, the formability of sheets is heavily dependent on the crystallographic texture and grain structure. For aluminium alloy sheets, the cold rolling texture has been characterized as the $\beta$ fibre (Hirsch \& Lücke, 1988), which is associated with plane strain deformation. However, the nonuniform deformation, mainly due to the substantial friction between rolls and sheet, could lead to the formation of pronounced shear textures in the surface layers of a rolled sheet. Therefore the surface layers show different behaviour from the plane-strain-deformed centre layer (Schoenfeld \& Asaro, 1996; Vasudévan et al., 1988).

We have measured the crystallographic texture of a $12.6 \mathrm{~mm}$-thick plate of AA7150 (86.28\% Al, 6.9\% Zn, $2.7 \%$ $\mathrm{Mg}, 2.5 \% \mathrm{Cu}, 1.5 \% \mathrm{Fe}, 0.12 \% \mathrm{Si}$ ) in the $\mathrm{T} 6$ condition (solution plus artificial ageing). Texture variations are known to exist across the thickness of such plates (Dutkiewicz \& Bonarski, 1997; Stelmukh, 2003), so in order to produce spatially homogeneous samples, five slices (corresponding to different depths) were cut by electro-discharge machining (EDM) from a $55 \times 92 \mathrm{~mm}$ plate. Subsequently, six $25 \mathrm{~mm}$-diameter discs were separated by EDM from each slice and stacked together with Super Glue, hence producing a fairly homogeneous cylindrical specimen of $12 \mathrm{~mm}$ height. The specimen corresponding to the mid-thickness of the original plate was measured on ENGIN-X using an instrument configuration and data analysis strategy identical to that for the copper specimen (gauge volume $6 \times 6 \times 4 \mathrm{~mm}$, incident beam divergence of $\sim 0.4 \times 0.5^{\circ}$, Euler goniometer for rotation, virtual detectors in $2 \times 5$ gridding scheme). In this case, TOF diffractograms were collected for 47 specimen orientations with counting times of 13 min per orientation, given the lower

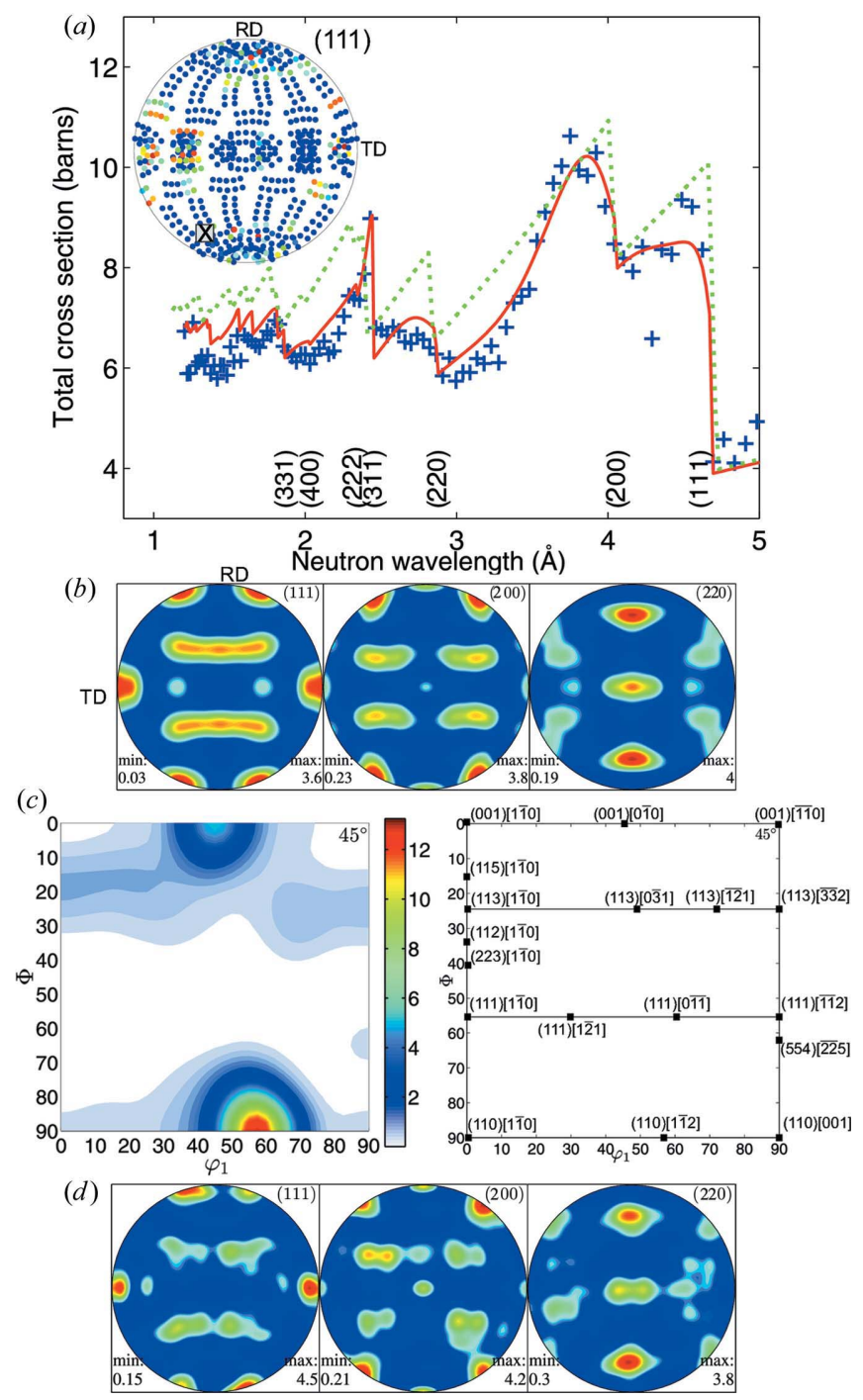

Figure 5

(a) Experimental (blue crosses) and calculated (red line) neutron total cross section of an aluminium (AA7150) rolled plate along the direction indicated as ' $X$ ' in the experimental pole figure of the inset. (b) Recalculated pole figures from the ODF calculated with MTEX from experimental pole figures such as that of the inset. (c) Cut of the ODF at $\varphi_{2}=45^{\circ}$, showing the main texture components of the material. $(d)$ Recalculated pole figures from companion X-ray diffraction experiments.' 
scattering power of $\mathrm{Al}$ as compared to $\mathrm{Cu}$. This scan yielded a total of 940 points for each experimental pole figure, as shown in the inset of Fig. 5(a) for the (111) pole figure. Again, the ODF of the material was calculated with $M T E X$ from the (111), (100), (110) and (311) experimental pole figures assuming triclinic sample symmetry. Fig. 5(b) shows recalculated pole figures, which are compared with the results obtained with laboratory X-rays $(d)$ on the same specimen, using the same experimental procedures and data analysis methods as for the steel specimen. Given the higher penetration of $\mathrm{Cu} K \alpha$ radiation on $\mathrm{Al}$ alloys, in between 100 and $200 \mu \mathrm{m}$, the averaging capabilities are better and the pole figures are measured with higher accuracy. Good qualitative and quantitative agreement is found between the pole figures obtained by the two techniques, yet some minor differences are observed, presumably due to some spatial texture inhomogeneity still present in the sample, coupled to differences in the actual volume gauged by the two techniques. Fig. 5(c) shows a section of the ODF, where two main components clearly emerge from the plot. These are a (110)[112] brass component characteristic of rolled aluminium, with a volume fraction of $30 \%$ of the crystallites, and a (001) [0 $\overline{1} 0]$ component with a $5 \%$ volume fraction. The volume fractions were computed by $M T E X$ using a $15^{\circ}$ radius sphere centred at each orientation.

The total cross section of the material was simultaneously measured during the experiment with the ENGIN-X transmission detector. Typical results are displayed in Fig. 5(a), for the direction identified in the experimental pole figure shown in the inset. The solid red curve shows calculations based on the experimentally determined ODF, which roughly captures the observed deviations from the isotropic case (green dashed curve).

3.3.1. Aluminium welding plates. Welding is a potentially beneficial fastening process for aircraft construction as it reduces weight and costs. However, for it to be seriously considered as an alternative to mechanical fasteners the interrelated problems of residual stress and distortion need to be addressed. In addition, welding introduces sharp spatial gradients in microstructure and crystallographic texture, which undoubtedly influence the integrity and performance of the weld and surrounding region. Texture and microstructure also impact on the capability of residual stress determination by diffraction-based techniques. Therefore, previous knowledge of the material texture is important for proper determination of residual stresses in aluminium alloy welds (Stelmukh, 2003). Variable polarity plasma arc (VPPA) welding is one method of joining aluminium alloys with potential application in the aerospace industry. To demonstrate the capability for nondestructive texture analysis on a small volume within a large object, we determined the ODF at two locations of VPPA-welded plates. The specimens were produced at Cranfield University Welding Research Group, UK, to be used as reference standards for residual stress measurements using neutron and synchrotron diffraction (ISO/TTA 3:2001, Polycrystalline Materials - Determination of Residual Stresses by Neutron Diffraction.). Two plates with
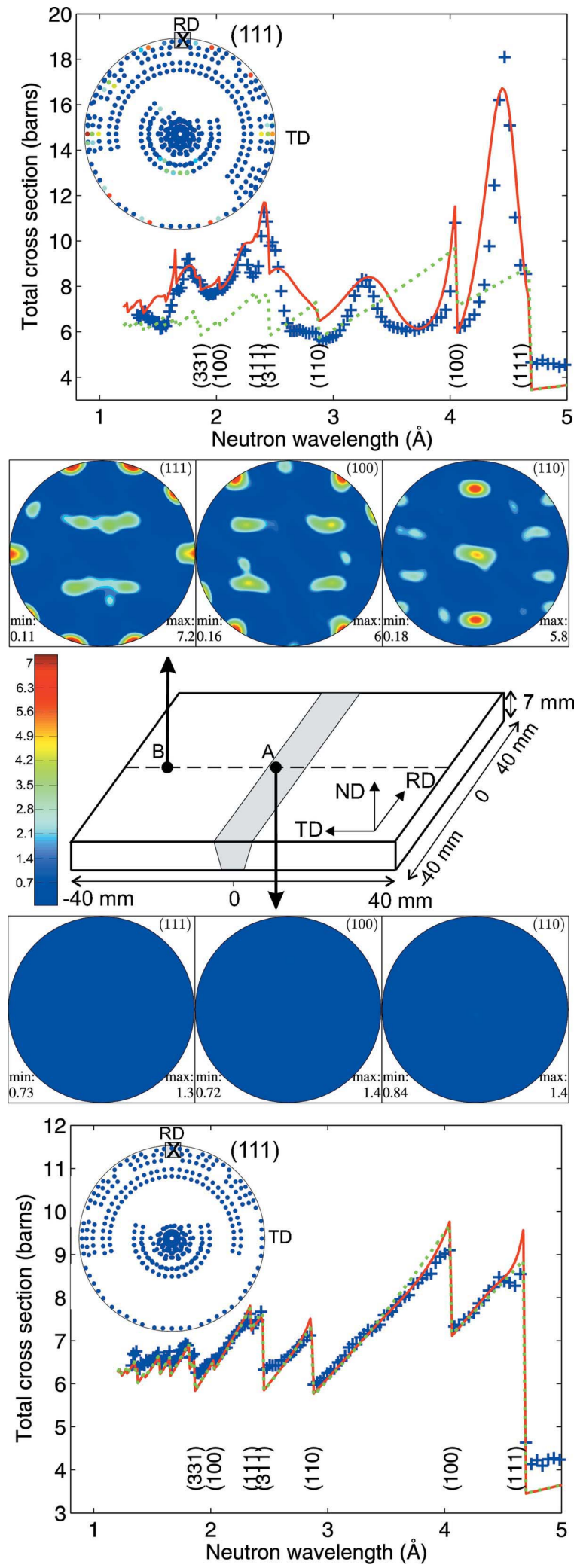

Figure 6

Texture changes in aluminium (AA2024) welded plates. Nondestructive measurements were performed at the weld centre (position A) and in the parent material (position B). The recalculated pole figures for those positions are markedly different. This is also reflected in the total cross section measured along the rolling direction at those locations. The material at the weld centre presents a nearly isotropic distribution of orientations. 
dimensions of $500 \times 186 \times 12 \mathrm{~mm}$ made of aluminium alloy 2024 conforming to aviation standards were joined along the rolling direction using an optimized VPPA procedure. The nominal composition of the alloy in wt \% was $4.35 \mathrm{Cu}, 1.50 \mathrm{Mg}$, $0.60 \mathrm{Mn}, 0.50 \mathrm{Fe}, 0.50 \mathrm{Si}, 0.25 \mathrm{Zn}, 0.20 \mathrm{Zr}, 0.15 \mathrm{Ti}$ and $0.10 \mathrm{Cr}$ ), and the original plates were in the T351 condition (solution heat treated, cold worked and naturally aged). After welding, the $12 \mathrm{~mm}$ plate was skimmed equally on both sides in $1 \mathrm{~mm}$ increments until the plate was $7 \mathrm{~mm}$ thick. Three nominally identical $80 \times 80 \mathrm{~mm}$ square samples were machined from this plate using EDM (Fig. 6, centre). The changes in microstructure and texture across the weld were investigated by Ganguly (2004). Ganguly reports electron backscatter diffraction (EBSD) pole figures for the material in the parent plate and fusion zone, obtained with a step size of $20 \mu \mathrm{m}$ over a $2 \times 2 \mathrm{~mm}$ grid. Here, we have measured pole figures using a $4 \times 4 \times 4 \mathrm{~mm}$ gauge volume on ENGIN-X at similar locations of the specimen, namely, at the weld centre (position A) and at a distance of $35 \mathrm{~mm}$ from it, in the parent material (position B). TOF diffractograms were recorded at 18 specimen orientations for position A and at 24 orientations for position B, with counting times of $\sim 17$ min per orientation. Rotation of the specimen was performed with the Cybaman manipulator, a three-axis goniometer supporting specimens of up to $20 \mathrm{~kg}$, fixed to a three-axis positioning table. After precise alignment of the specimen, the region to be investigated was brought to the centre of the diffractometer by translations with the positioning table. Fig. 6 shows recalculated pole figures (in the same colour scale) for the two locations from the ODF determined with MTEX from the (111), (100), (110) and (311) experimental pole figures using a $2 \times 5$ gridding scheme and considering triclinic sample symmetry. The weld texture is clearly different from the parent plate texture. The strong texture found in the parent plate becomes an almost perfectly random distribution at the weld centre. The texture obtained for the parent plate differs considerably from the one previously reported for the sample (Ganguly et al., 2008) but presents the same general features of the texture expected for an f.c.c. rolling metal (Kocks, 2000). The ODF differences clearly manifest themselves in the total cross section curves measured along the rolling directions at locations $\mathrm{A}$ and $\mathrm{B}$ (Fig. 6). A nearly isotropic total cross section is measured at the weld centre, whilst large deviations from such an ideal case are observed in the parent plate, which are well captured by the calculation based on the refined ODF (red line). Such good agreement confirms the soundness of the performed texture analysis based on incomplete pole figures, as shown in the insets.

\subsection{Zr2.5\% Nb pressure tubes}

Owing to their good mechanical strength and high corrosion and creep resistance, combined with excellent neutronic properties, Zr-based alloys have been selected as the materials of choice for nuclear fuel cladding worldwide (Zircaloy-4 and 2) and for pressure tubes in CANDU and RBMK reactors $(\mathrm{Zr} 2.5 \% \mathrm{Nb}$ and $\mathrm{Zr} 1 \% \mathrm{Nb})$. The crystallographic texture of tubing is a parameter carefully specified by designers owing to its impact on in-reactor performance, mostly because it influences irradiation growth and hydrogen-related degradation mechanisms. Here we have measured the texture of pressure tubes used in a CANDU nuclear power plant. The tubes are $\sim 4 \mathrm{~mm}$ thick and $\sim 6 \mathrm{~m}$ long, with a $\sim 100 \mathrm{~mm}$ diameter. Starting from $\mathrm{Zr} 2.5 \% \mathrm{Nb}$ billets forged at $\sim 1073 \mathrm{~K}$, the fabrication route of pressure tubes included extrusion at $\sim 1073 \mathrm{~K}$, followed by cold drawing to $\sim 28 \%$ strain and autoclaving at $673 \mathrm{~K}$ for $24 \mathrm{~h}$ (Holt, 2008). The microstructure of the pressure tube consists of $\alpha$ grains up to about $10 \mu \mathrm{m}$ long, $1 \mu \mathrm{m}$ wide and $0.5 \mu \mathrm{m}$ thick, which have an h.c.p. crystal structure containing between 0.6 and $1 \mathrm{wt} \% \mathrm{Nb}$, surrounded by a grain boundary network of $\mathrm{Nb}$-stabilized $\beta$ - Zr.

Small sections having dimensions of $\sim 10 \times 20 \mathrm{~mm}$ along the axial and rolling directions, respectively, were machined out of experimental $\mathrm{Zr} 2.5 \% \mathrm{Nb}$ pressure tubes. The samples correspond to different stages of a slightly modified manufacturing route being developed at Comisión Nacional de Energía Atómica, Argentina, which uses a pilger-type cold rolling stage instead of the cold drawing stage (Banchik, 2009). The first specimen is from an extruded tube, the starting material for the process. The second specimen corresponds to the same material after $28 \%$ cold rolling. The third specimen is from a finished tube, obtained after a $24 \mathrm{~h}$ heat treatment at $673 \mathrm{~K}$ of the cold-rolled material.

The specimens were measured on ENGIN-X using a gauge volume of $6 \times 4 \times 4 \mathrm{~mm}$, an incident beam divergence optimized for texture measurement, of $\sim 0.7 \times 0.8^{\circ}$, and a counting time of 6.5 min per specimen orientation. TOF diffractograms were recorded at only four sample orientations, carefully chosen to cover the most relevant parts of the pressure tube pole figures. The first orientation was that shown in Fig. 1(a), with the hoop direction along the south bank and the radial direction along the north bank. Two additional orientations corresponded to 15 and $30^{\circ}$ rotations around the vertical axis, respectively, and the third one to a $90^{\circ}$ rotation around the horizontal (radial) axis. These four orientations resulted in a total time of $0.5 \mathrm{~h}$ per specimen investigated. Data analysis was performed with virtual detectors in an $8 \times 5$ gridding scheme, which results in exploring 80 points of the pole figure from a single specimen orientation with a $\sim 2 \times 8^{\circ}$ angular window per virtual detector. The resulting coverage of the pole figure is shown in Fig. 7(a), together with the results for the (0002) pole figure measured for the three specimens for directions contained in the radial-hoop plane.

The ODF of the material was calculated with MTEX from

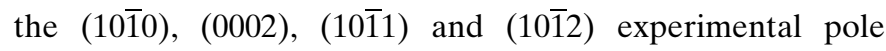
figures, considering triclinic sample symmetry. Recalculated pole figures for the three specimens are shown in Figs. 7(b), $7(c)$ and $7(d)$. The final (heat-treated) specimen displays the typical features observed in $\mathrm{Zr} 2.5 \% \mathrm{Nb}$ pressure tubes (Holt, 2008) and shows excellent agreement with results obtained by other techniques, including both constant-wavelength neutron and X-ray diffraction (Santisteban, Malamud et al., 2014), and synchrotron X-ray diffraction in transmission geometry (Vicente Alvarez et al., 2011). The (0002) basal planes present 
a pole along the hoop direction, which elongates towards the radial direction, while the prismatic $(10 \overline{1} 0)$ planes show strong pole densities along the axial direction. Most grains have their $c$ axis pointing in the hoop direction and their [10 $\overline{1} 0$ ] axis parallel to the axial direction. However, there are also a great number of grains with their [10 $\overline{1} 0]$ axis parallel to the axial direction but with their $c$ axis rotated by up to $45^{\circ}$ from the hoop direction. These grains are responsible for the elongated structure observed between the hoop and radial direction in the (0001) pole figure. By contrast to steel and aluminium alloys, we see here that no dramatic changes in texture occur during thermal treatment of Zr-based alloys; hence, the deformation texture is essentially preserved after annealing. However, some small texture changes are observed during
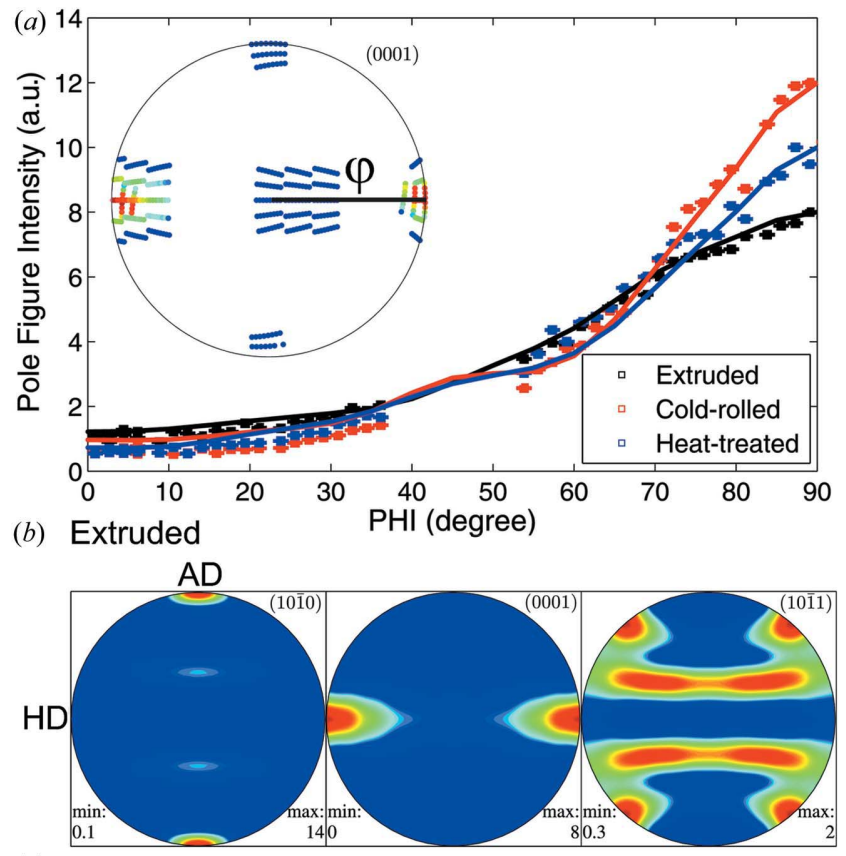

(c) Cold rolled

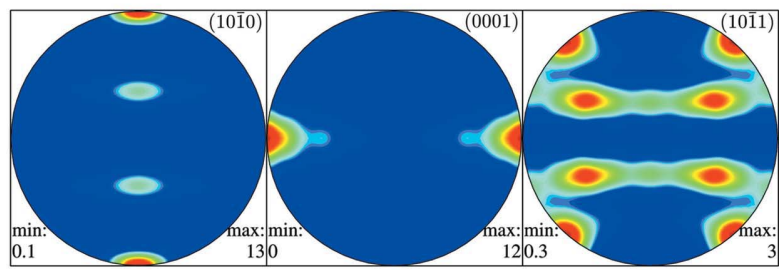

(d) Heat treated

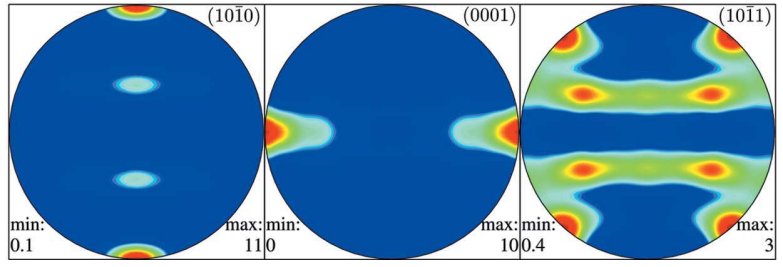

Figure 7

ODF of $\mathrm{Zr} 2.5 \% \mathrm{Nb}$ pressure tubes at different stages of manufacturing. The recalculated pole figures shown in $(b),(c)$ and $(d)$ were produced from experimental pole figures covering the orientations shown in the inset of $(a)$. This figure also shows details of the experimental pole figure along the hoop-radial line for three manufacturing stages.
Table 1

Kearns factors for the principal directions of the $\mathrm{Zr} 2.5 \% \mathrm{Nb}$ pressure tubes at different stages of the manufacturing process calculated from the ODFs obtained in the present work and from other neutron diffractometers.

\begin{tabular}{llll}
\hline & \multicolumn{3}{l}{ Kearns factor $( \pm 0.02)$} \\
\cline { 2 - 4 } Pressure tube manufacturing stage & Hoop & Radial & Axial \\
\hline Extruded & 0.564 & 0.361 & 0.073 \\
Cold rolled & 0.618 & 0.288 & 0.091 \\
Cold rolled + heat treated (CR+HT) & 0.556 & 0.335 & 0.108 \\
CR+HT other instruments & & & \\
Kowari & 0.546 & 0.356 & 0.094 \\
SKAT & 0.564 & 0.344 & 0.09 \\
\hline
\end{tabular}

manufacturing. In the experimental pole figure, cold rolling produces a drop of $\sim 30 \%$ in the number of crystals with $c$ axes along the hoop direction $\left(\varphi=90^{\circ}\right)$ and an increase in those with $c$ axes at angles $\varphi<60^{\circ}$. The refined ODFs effectively capture the small differences between specimens, as shown by the solid lines in Fig. $7(a)$ and by the different maximum intensities in the recalculated pole figures. Cold rolling also produces a $\sim 10 \%$ increase in the sharp axial (1010) pole, followed by a $\sim 20 \%$ decrease after heat treatment.

In manufacturing, the texture of products made of hexagonal (h.c.p.) materials is specified through Kearns factors (Kearns, 1965). These parameters assess the proportion of unit cells having their hexagonal $c$ axes projected along the three principal axes of the specimen, as given by equation (8). For tubing, Kearns factors are calculated for the hoop, radial and axial directions $\left(f_{\mathrm{H}}, f_{\mathrm{R}}\right.$ and $f_{\mathrm{A}}$, respectively). The calculated Kearns factors are shown in Table 1. For the final tube, we have also included results obtained at two other neutron diffractometers, SKAT at JINR, Russia (Ullemeyer et al., 1998), and Kowari, at OPAL, Australia (Brule \& Kirstein, 2006). Very good quantitative agreement is found between techniques.

The through-thickness variation of crystallographic texture was investigated on ENGIN-X using a $30 \mathrm{~cm}$-long section of a commercial pressure tube, with a spatial resolution of $0.5 \mathrm{~mm}$. After careful alignment of the specimen, TOF diffractograms were measured at seven through-thickness depths by bringing the region of interest of the tube into the centre of the diffractometer using the positioning table. The experiment was originally designed to determine residual stresses within the tube, so the incident beam divergence was tight, $0.4 \times 0.5^{\circ}$ (horizontal $\times$ vertical), and diffractograms were measured for only two specimen orientations. For the first orientation (Fig. 1a) we used a gauge volume of $0.5 \times 0.5 \mathrm{~mm}$ in the (horizontal) diffraction plane and $11 \mathrm{~mm}$ in the vertical direction (normal to the page), as allowed by the axial symmetry of the tube. This resulted in counting times of 40 min per point. In the second orientation the tube was positioned horizontally, with the axial direction measured by the north bank and the radial direction by the south bank, whilst the hoop direction was along the vertical. In this configuration, the length of the gauge volume in the vertical direction was reduced to $5 \mathrm{~mm}$, which resulted in rather long 
counting times of $\sim 80 \mathrm{~min}$ per point. Fig. $8(a)$ shows the variation in intensity of the 0002 reflection registered along the tube hoop direction, where a clear decrease in the signal is observed when approaching the tube outer surface. This agrees with previous results presented by Santisteban et al. (2012), obtained by energy-resolved neutron radiography, a novel technique that is finding an increasing number of applications (Strobl, 2013). Experimental and calculated total cross section values for the tube have been presented by Santisteban et al. (2012). For such small gauge volumes, the statistical quality collected for neutron strain scanning is not good enough for texture analysis. So, in order to perform the proposed texture analysis, the data were grouped into three different regions: inner, central and outer zones, as indicated in the figure. Diffractograms collected within a region were summed, to be subsequently analysed with virtual detectors in a $5 \times 5$ gridding scheme. This results in 50 points of the pole figure from each specimen orientation, with a $\sim 3 \times 8^{\circ}$ angular window per virtual detector. Although it is based on only two orientations, the pole figure still captures the most important specimen orientations, i.e. the axial direction containing the

(a)

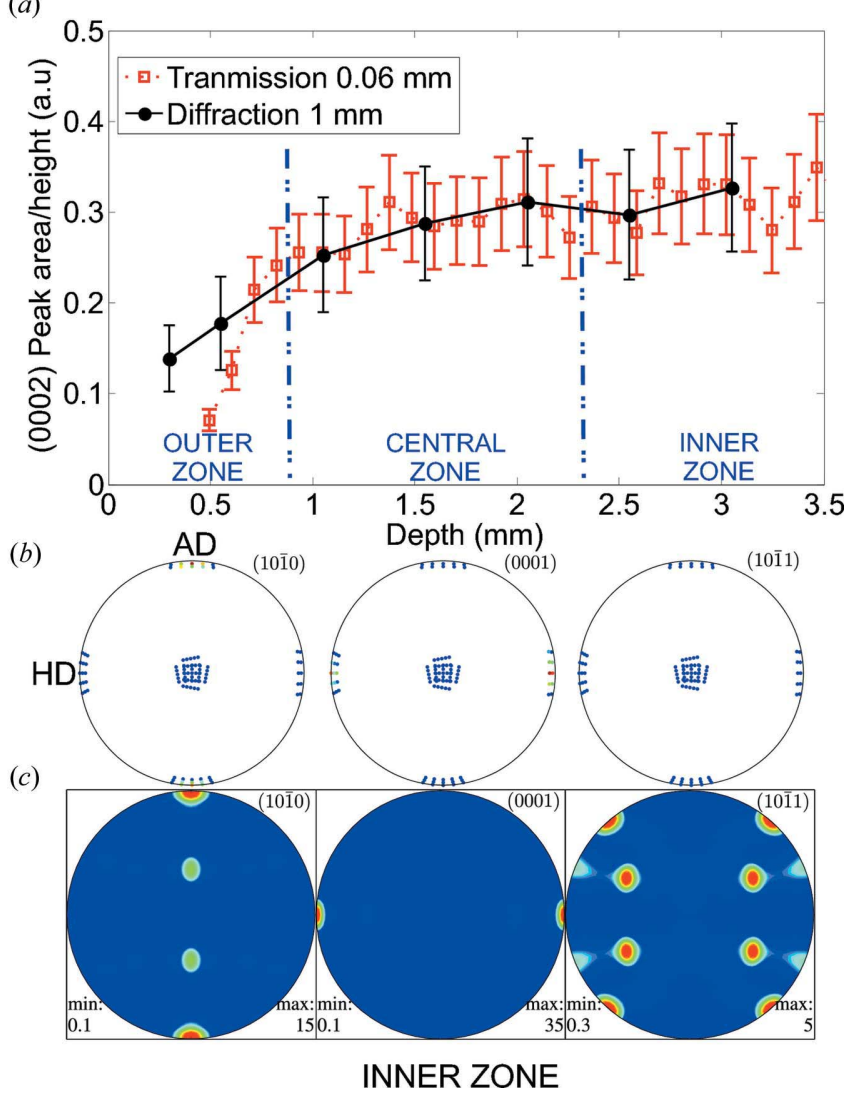

Figure 8

(a) Change in integrated area of the 0002 reflection registered along the tube hoop direction of commercial $\mathrm{Zr} 2.5 \% \mathrm{Nb}$ pressure tube (circles), compared with results obtained by energy-resolved neutron radiography (squares) (Santisteban et al., 2006). (b) Incomplete experimental pole figures restricted to narrow regions around the principal axes of the tube (hoop, radial, axial). (c) Recalculated pole figures for the tube central region obtained from the ODF calculated with MTEX from the (1010), (0002), (10 $\overline{1} 1)$ and $(10 \overline{1} 2)$ experimental pole figures.
Table 2

Kearns factors for the principal directions of the $\mathrm{Zr} 2.5 \% \mathrm{Nb}$ pressure tubes at different depths of a commercial $\mathrm{Zr} 2.5 \% \mathrm{Nb}$ pressure tube calculated from the ODFs obtained from incomplete pole figures.

\begin{tabular}{llll}
\hline & \multicolumn{2}{l}{ Kearns factor $( \pm 0.02)$} \\
\cline { 2 - 4 } Pressure tube depth & Hoop & Radial & Axial \\
\hline Outer region & 0.537 & 0.333 & 0.126 \\
Central region & 0.544 & 0.346 & 0.101 \\
Inner region & 0.553 & 0.335 & 0.109 \\
\hline
\end{tabular}

(1010) pole and the hoop direction containing the (0002) pole, as shown in Fig. 8(b). Hence, the ODF was calculated with

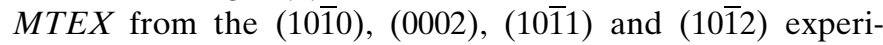
mental pole figures, considering triclinic sample symmetry. As observed in Fig. 8(c) for the central region, this results in reasonable recalculated pole figures, from which Kearns factors have been calculated for the three regions. The results listed in Table 2 show reasonable values that are within a typical \pm 0.02 uncertainty of those listed in Table 1 .

\section{Discussion}

In this work we have successfully used ENGIN-X, a TOF neutron strain scanner, to perform texture analyses on a number of metallic specimens. Table 3 summarizes the experimental arrangements, data analysis parameters and geometric dimensions of all specimens investigated. The method used here was validated by accompanying texture analysis using other experimental techniques. The main strength of the proposed method is its ability to nondestructively measure the ODF of a small volume of material (from $\sim 1 \times 1 \times 1 \mathrm{~mm}$ to $\sim 6 \times 6 \times 6 \mathrm{~mm}$ ) contained within a large object, as demonstrated here by experiments on AA2024 welded plates and on commercial $\mathrm{Zr} 2.5 \% \mathrm{Nb}$ pressure tubes.

The method is based on splitting ENGIN-X detection banks into a number of smaller virtual detectors of sharper angular resolution, and defining the ODF of the material from a number of incomplete experimental pole figures using the MTEX toolbox.

Determination of an unknown ODF requires complete coverage of the $\mathrm{SO}(2)$ pole figure orientation space with a very fine angular grid. In practice, the adopted grid must be compatible with the angular resolving power of the texture diffractometer. This parameter is usually described by the instrumental transparency function, $T(\alpha, \beta)$, representing the actual resolution available to determine a pole figure (Moras et al., 2000). Hence, $T(\alpha, \beta)$ defines the size, shape and sharpness of the angular element $(\Delta \Omega=\sin \alpha \mathrm{d} \alpha \mathrm{d} \beta)$ used for building the experimental pole figures. Transparency functions of commercially available X-ray texture goniometers have half-widths of the order of several degrees, yet the details of $T(\alpha, \beta)$ depend on the spectral distribution of the source, the diffracting or Bragg angle, and the position and dimensions of the entrance and exit slits, source, and detector (Moras et al., 2000). In conventional X-ray reflection geometry, the halfwidth of the transparency function in the $\alpha$ and $\beta$ directions can be effectively characterized by $\alpha=\alpha^{90^{\circ}} / \sin \theta_{\mathrm{B}}$ and 
Table 3

Details of geometric dimensions of all specimens investigated in the present work.

The table lists also the experimental arrangements and data analysis parameters used for texture analysis for each of the studies.

\begin{tabular}{|c|c|c|c|c|c|c|c|c|c|}
\hline Sample & $\begin{array}{l}\text { Crystal } \\
\text { structure }\end{array}$ & $\begin{array}{l}\text { Sample } \\
\text { shape }\end{array}$ & $\begin{array}{l}\text { Sample } \\
\text { dimensions }(\mathrm{mm})\end{array}$ & $\begin{array}{l}\text { Gauge volume } \\
\text { dimensions }(\mathrm{mm})\end{array}$ & $\begin{array}{l}\text { Incident } \\
\text { divergence }\left({ }^{\circ}\right)\end{array}$ & $\begin{array}{l}\text { Sample } \\
\text { orientations }\end{array}$ & $\begin{array}{l}\text { Counting } \\
(\mu \mathrm{A} \mathrm{h})\end{array}$ & $\begin{array}{l}\text { Gridding } \\
\text { scheme }\end{array}$ & $\begin{array}{l}\text { Virtual detector } \\
\text { solid angle }\left({ }^{\circ}\right)\end{array}$ \\
\hline Low-carbon steel & B.c.c. & Cylinder & $\varnothing: 10 ;$ h: 8 & $6 \times 4 \times 4$ & $0.7 \times 0.8$ & 31 & 3.5 & $2 \times 5$ & $8 \times 8$ \\
\hline Copper & F.c.c. & Irregular & $20 \times 20 \times 10$ & $6 \times 6 \times 4$ & $0.4 \times 0.5$ & 57 & 7 & $2 \times 5$ & $8 \times 8$ \\
\hline AA7150 & F.c.c. & Cylinder & $\varnothing: 25 ; \mathrm{h}: 12$ & $6 \times 6 \times 4$ & $0.4 \times 0.5$ & 47 & 13 & $2 \times 5$ & $8 \times 8$ \\
\hline AA2024 (PM) & F.c.c. & Plate & $80 \times 80 \times 7$ & $4 \times 4 \times 4$ & $0.4 \times 0.5$ & 24 & 25 & $2 \times 5$ & $8 \times 8$ \\
\hline AA2024 (FZ) & F.c.c. & Plate & $80 \times 80 \times 7$ & $4 \times 4 \times 4$ & $0.4 \times 0.5$ & 18 & 25 & $2 \times 5$ & $8 \times 8$ \\
\hline $\mathrm{Zr} 2.5 \% \mathrm{Nb}$ experimental & H.c.p. & Iregular & $10 \times 20 \times 4$ & $6 \times 6 \times 4$ & $0.7 \times 0.8$ & 4 & 3.5 & $8 \times 5$ & $2 \times 8$ \\
\hline $\mathrm{Zr} 2.5 \% \mathrm{Nb}$ commercial & H.c.p. & Tube & $\varnothing: 10 ; \mathrm{h}: 300 \mathrm{t}: 4$ & $0.5 \times 0.5 \times 5$ & $0.4 \times 0.5$ & 2 & 60 & $5 \times 5$ & $3 \times 8$ \\
\hline
\end{tabular}

$\beta=\omega / \sin \alpha$; with $\Delta \alpha^{90^{\circ}}$ and $\Delta \omega$ the width values extrapolated, respectively, for $\theta_{\mathrm{B}}=90^{\circ}$ and $\alpha=90^{\circ}, \alpha$ being the angle between the specimen normal and the impulse exchange vector (Moras et al., 2000). By contrast, in TOF neutron strain scanners the Bragg angle is approximately fixed at $\theta_{\mathrm{B}} \simeq 45^{\circ}$ and the $\alpha$ angle plays essentially no role in this instrumental geometry. Owing to the polychromatic incident beam, the transparency function becomes considerably more complex, and no specific analysis of $T(\alpha, \beta)$ has yet been presented in the open literature. For the method proposed here, $T(\alpha, \beta)$ is highly dependent on the strategy adopted in defining the virtual detectors, as exemplified in Fig. 1 for different gridding schemes. Hence, the ENGIN-X angular resolution could in principle be tailored to the problem being investigated, as observed by the different virtual detector solid angles listed in Table 3. There is, however, a physical limit to $T(\alpha, \beta)$, determined by the geometry of the individual detector element, and to a lesser extent by the radial collimator and incident beam slit apertures chosen for the study. The $3 \times 196 \mathrm{~mm}$ scintillator at $\sim 1.5 \mathrm{~m}$ from sample results in an anisotropic angular element of $\sim 0.1 \times 7.5^{\circ}$, which has to be convoluted to the incident and scattered beam divergences in the horizontal and vertical planes. The considerable height of the detector element gives a very loose definition of the diffracted beam in the direction normal to the diffraction plane, little affected by the opening of the incident slits (typically between 0.3 and $0.8^{\circ}$ ). Hence, in the vertical direction the transparency function is essentially flat over $\sim 7.5^{\circ}$, with slightly blurred edges over a range of $\sim 0.5^{\circ}$, producing an ultimate resolving power of $-0.1 \times 8^{\circ}$. After grouping the detectors according to the selected gridding scheme, the transparency function of the virtual detector will correspond to the convolution of the transparency function of the individual elements.

Optimal definition of the virtual detectors' gridding scheme must also consider the details of the algorithm used to extract the orientation distribution function. In the $M T E X$ algorithm the ODF of the crystallites is approximated as a superposition of a large number of radially symmetric bell-shaped model ODFs. Each of these unimodal components of the ODF is centred at a discrete location in the $\mathrm{SO}(3)$ Euler orientation space and depends only on the (angular) distance to that centre. The number, position and width of these components is defined by the angular resolution adopted to describe the regular $\mathrm{SO}(3)$ grid, which must be compatible with the experimental $\mathrm{SO}(2)$ grid used to produce the pole figures. Hence the MTEX method somehow assumes that pole figures are recorded with a detector covering an isotropic angular element in the $(\alpha, \beta)$ grid. The transparency function of ENGIN-X can be made approximately isotropic by adopting the $2 \times 5$ gridding scheme, which gives a virtual detector covering a solid angle of $\Delta \Omega \simeq 8 \times 8^{\circ}$. This justifies the use of such a gridding scheme in most of the experiments described here. This angular resolution is sharper than those for other TOF texture diffractometers such as HIPPO, which has an optimal texture resolution of $\sim 12^{\circ}$ as a result of its detector geometry (Matthies et al., 2005).

The angular resolution $\Delta \simeq 8^{\circ}$ dictates the FWHM of the unimodal components used in the MTEX algorithm to represent the ODF and, indirectly, the discretization of the $\mathrm{SO}(3)$ Euler space. This is because effective description of an unknown and reasonably smooth ODF by a linear combination of unimodal components requires that such components be uniformly distributed in the orientation space with a pitch of $\sim(2 / 3) \Delta$ (Hielscher \& Schaeben, 2008). Hence, pole figures measured with a $2 \times 5$ gridding scheme were analysed with MTEX using unimodal components described by a de la Vallée Poussin kernel having an FWHM of $8^{\circ}$ (Hielscher \& Schaeben, 2008), distributed on a regular $5 \times 5 \times 5^{\circ}$ discretization of the $\mathrm{SO}(3)$ Euler space.

Complete pole figure coverage with $\sim 8^{\circ}$ resolution on a regular $(\alpha, \beta)$ grid can be attained in ENGIN-X with $N_{\text {orient }} \simeq$ 45 specimen orientations using a $2 \times 5$ gridding scheme of the detection banks, as exemplified by the inset of Fig. 5(a). However, in normal practice experimental time is limited, and several strategies must be sought in order to minimize the experimental time required for texture analysis. Use of an (approximate) equal-area $\mathrm{SO}(2)$ grid would reduce the number of sample orientations necessary to cover the pole figures to $N_{\text {orient }} \simeq 30$ (Matthies \& Wenk, 1992). Details of the required specimen orientation using an Euler goniometer in ENGIN-X and the resulting pole figure coverage are shown in Appendix $A$. Symmetry operations of the sample and the crystal system reduce the number of non-equivalent orientations in the $\mathrm{SO}(2)$ grid. The (statistical) sample symmetry is directly related to the symmetry of the preceding deformation, so it can considerably reduce the number of explored specimen orientations when the thermomechanical history of the sample is known with confidence. 
The wavelength range used in the experiment defines the number of pole figures available for the ODF analysis. ENGIN-X is at the end of a curved guide looking at a liquid methane moderator, providing a maximum flux for $d$ spacings of $\sim 1.4 \AA$ and falling sharply for values lower than $\sim 0.6 \AA$ (Fig. 7c of Santisteban et al., 2006). The available wavelength range can be selected by means of choppers that alter the rate and TOF width of the incident neutron pulse. Pulse rates of 25 and $16.67 \mathrm{~Hz}$ offer $d$-spacing ranges of $\Delta d \simeq 1.6 \AA$ and $\Delta d \simeq$ $2.4 \AA$, respectively. A $25 \mathrm{~Hz}$ pulse rate would be optimal for combined texture + strain analysis, as it offers access to $\sim 10$ reflections for the most common engineering materials. However, count rates fall sharply for $d$ spacings lower than $\sim 1 \AA$ (Fig. $8 a$ of Santisteban et al., 2006), so in a time-optimized experiment the actual number of peaks included in the ODF analysis may be half of this number, owing to poor statistics for the higher-order reflections. In most of the present experiments, we used a $16.67 \mathrm{~Hz}$ pulse rate in order to access a wide wavelength range for simultaneous total cross section measurements.

The individual counting time required per specimen orientation can be minimized by increasing the incident beam divergence, as done here for the experiments on steel and on the experimental $\mathrm{Zr} 2.5 \% \mathrm{Nb}$ pressure tubes. However, a higher divergence also increases the peak width, affecting the possibility to perform simultaneous strain analysis. Hence, depending on the case, the actual value of the incident divergence should emerge as a compromise between the optimization of the two techniques. As seen in Table 3, two incident divergences were used in this work: one optimized for strain scanning $\left(0.4 \times 0.5^{\circ}\right)$ and one optimized for texture measurements $\left(0.7 \times 0.8^{\circ}\right)$. Besides the incident divergence, the counting time per specimen orientation depends directly on the accelerator proton current used in the $50 \mathrm{~Hz}$ spallation process producing the neutron pulses and on the actual fraction of these pulses that arrive at the sample. The proton current is usually stable but it may suffer temporary reductions in intensity, and several pulse rates are available on ENGIN-X. These rates were initially $12.5,16.7$ and $25 \mathrm{~Hz}$, and were changed to $4 / 5$ of these values after a second target station started operation at ISIS. As different pulse rates and proton currents were used through these experiments, Table 3 lists the counting time per orientation in terms of the 'effective' integrated proton current $(\mu \mathrm{A} \mathrm{h})$, which is proportional to the number of neutrons that have actually arrived at the sample.

From the previous analysis, the optimal strategy for texture analysis of a specimen having an unknown ODF would use a $25 \mathrm{~Hz}$ pulsed neutron beam with a relaxed incident divergence, to measure $\sim 30$ specimen orientations chosen to produce an approximately hexagonal pole figure grid, as described in Appendix $A$. This would produce between four and six full pole figures with $\sim 8^{\circ}$ resolution, which is optimal for ODF analysis with MTEX using a conventional $5 \times 5 \times 5^{\circ}$ grid of the Euler space. For a gauge volume of $\sim 5 \times 5 \times 5 \mathrm{~mm}$ and low beam attenuation, the counting times per orientation are estimated to be about $4 \mathrm{~min}$ for steel and bronzes, and
$8 \mathrm{~min}$ for aluminium and zirconium alloys, resulting in total times for texture analysis of 2.5 and $5 \mathrm{~h}$, respectively.

On the other hand, in many engineering applications prior knowledge exists about the main features of the material ODF. In such cases, the interest may be centred in determining volume fractions of specific texture components, or quantifying magnitudes derived from the ODF, such as Kearns factors in hexagonal materials. When this is the case, both the instrument resolution and the pole figure coverage can be tailored to the specific problem, in order to produce faster and more detailed texture analyses. This was done here in the analysis of the experimental $\mathrm{Zr} 2.5 \% \mathrm{Nb}$ pressure tubes at different stages of manufacturing, where a finer $2 \times 8^{\circ}$ resolution was used by means of an $8 \times 5$ gridding scheme (Table 3). In the experimental arrangement, the specimen was oriented so that a $\sim 2^{\circ}$ resolution was used to explore the hoop-radial line of the pole figure (Fig. 1a), in order to precisely resolve the variation that occurs along this line in the (0002) pole figure (Fig. 7). Measurements at only four specimen orientations resulted in a total counting time of $\sim 30$ min per specimen, yet still allowed a combined texture, stress and dislocation density analysis to be performed (Santisteban, Vizcaíno et al., 2014). Pole figures and Kearns factors obtained for the final product agreed well with those from other instruments, as described by Santisteban, Malamud et al. (2014).

Finally, in cases where prior knowledge of the ODF is uncertain or not available and experimental time is scarce, a sparse coarse grid can be used in combination with transmission measurements. In this work, such a strategy proved useful in the analysis of the AA2024 aluminium welds (Fig. 6), where good agreement between measured and calculated total cross section values supported the ODFs determined in the present experiments, which contradicted those previously obtained by EBSD on much smaller sample volumes. In extreme cases, valuable texture information can still be obtained by applying the method described here to experiments originally performed for standard strain analysis (which measures only solid angles around the specimen principal axes). This was demonstrated here in the study of texture gradients on commercial pressure tubes (Fig. 8).

As a final comment, we note that the neutron beam attenuation was rather weak within all specimens investigated; hence no corrections were needed to produce the experimental pole figures. In cases where such effects proved important, the SSCANSS software (James et al., 2004), originally developed for ENGIN-X, can be used to calculate the beam attenuation for specimens of arbitrary geometry.

\section{Conclusions}

Nondestructive texture measurements with a typical spatial resolution of $\sim 4 \times 4 \times 4 \mathrm{~mm}$ and angular resolution of $\sim 8^{\circ}$ have been performed on a variety of metallic specimens using ENGIN-X, a time-of-flight neutron strain scanner at the ISIS facility, UK. The materials investigated included examples of interest to the aerospace industry (aluminium alloys), auto- 
Table 4

Euler angles used for the pole figure coverage shown in Fig. 9(b).

\begin{tabular}{|c|c|c|}
\hline$\varphi_{1}\left(^{\circ}\right)$ & $\Phi\left({ }^{\circ}\right)$ & $\varphi_{2}\left(^{\circ}\right)$ \\
\hline 30 & 90 & 0 \\
\hline 30 & 90 & 30 \\
\hline 30 & 90 & 60 \\
\hline 30 & 90 & 90 \\
\hline 30 & 90 & 120 \\
\hline 30 & 90 & 150 \\
\hline 30 & 90 & 180 \\
\hline 30 & 90 & 210 \\
\hline 30 & 90 & 240 \\
\hline 30 & 90 & 270 \\
\hline 30 & 90 & 300 \\
\hline 30 & 90 & 330 \\
\hline 41 & 90 & 0 \\
\hline 45 & 90 & 0 \\
\hline 45 & 35 & 0 \\
\hline 45 & 35 & 20 \\
\hline 45 & 35 & 40 \\
\hline 45 & 35 & 60 \\
\hline 45 & 35 & 80 \\
\hline 45 & 35 & 100 \\
\hline 45 & 35 & 120 \\
\hline 45 & 35 & 140 \\
\hline 45 & 35 & 160 \\
\hline 45 & 35 & 180 \\
\hline 45 & 35 & 200 \\
\hline 45 & 35 & 220 \\
\hline 45 & 35 & 240 \\
\hline 45 & 35 & 260 \\
\hline 45 & 35 & 280 \\
\hline 45 & 35 & 300 \\
\hline 45 & 35 & 320 \\
\hline 45 & 35 & 340 \\
\hline 49 & 90 & 0 \\
\hline 135 & 55 & 10 \\
\hline 135 & 55 & 40 \\
\hline 135 & 55 & 70 \\
\hline 135 & 55 & 100 \\
\hline 135 & 55 & 130 \\
\hline 135 & 55 & 160 \\
\hline 135 & 55 & 190 \\
\hline 135 & 55 & 220 \\
\hline 135 & 55 & 250 \\
\hline 135 & 55 & 280 \\
\hline 135 & 55 & 310 \\
\hline 135 & 55 & 340 \\
\hline
\end{tabular}

motive industry (low-carbon steels), nuclear industry (zirconium alloys) and cultural heritage (copper alloys). Several (incomplete) experimental pole figures are simultaneously produced after splitting the two detection banks into a number of smaller virtual detectors. The material ODF is obtained from these pole figures using the $M T E X$ algorithm: especially suited for sharp textures and pole figures measured on scattered specimen directions. All the operations involved in the texture analysis presented here (splitting of detection banks and producing the associated diffractograms, least-squares peak fitting, pole figure creation, ODF analysis, total cross section calculation) have been implemented in a freely available MATLAB library $(N y R T e x)$.

From the examples analysed here, the following conclusions can be drawn:

(i) The optimal experimental arrangement for characterizing a material with an unknown ODF consists of splitting each diffraction bank into ten virtual detectors $(5$ rows $\times 2$ columns) and producing complete pole figures by measuring TOF diffractograms for $\sim 30$ orientations of the specimen. For a gauge volume of $\sim 4 \times 4 \times 4 \mathrm{~mm}$, counting times per texture analysis are in the range of 2.5 and $5 \mathrm{~h}$, depending on the material investigated.

(ii) The spatial resolution can be improved up to $\sim 1 \mathrm{~mm}$ and the angular resolution up to $\sim 2^{\circ}$ for one of the Euler angles. This can be exploited to investigate fine features in engineering applications where the overall shape of the ODF of the specimens is known, as shown here for $\mathrm{Zr} 2.5 \% \mathrm{Nb}$ pressure tubes at different stages of manufacturing. In such cases, pole figure coverage can be easily focused on regions of interest, considerably reducing experimental times.

(iii) For unknown specimens where time constraints limit measurements to rather incomplete pole figures, the soundness of the resulting ODF can be assessed by performing fast and simple transmission experiments with existing instrumentation. Comparison between calculated and experimental total neutron cross sections along different directions of the specimen would show possible shortcomings of the proposed ODF.

\section{APPENDIX $\boldsymbol{A}$}

In traditional X-ray texture analysis pole figures are scanned using the Schulz reflection method with equal angular steps for $\alpha$ and $\beta$ (typically $5 \times 5^{\circ}$ ). In ENGIN-X, rather

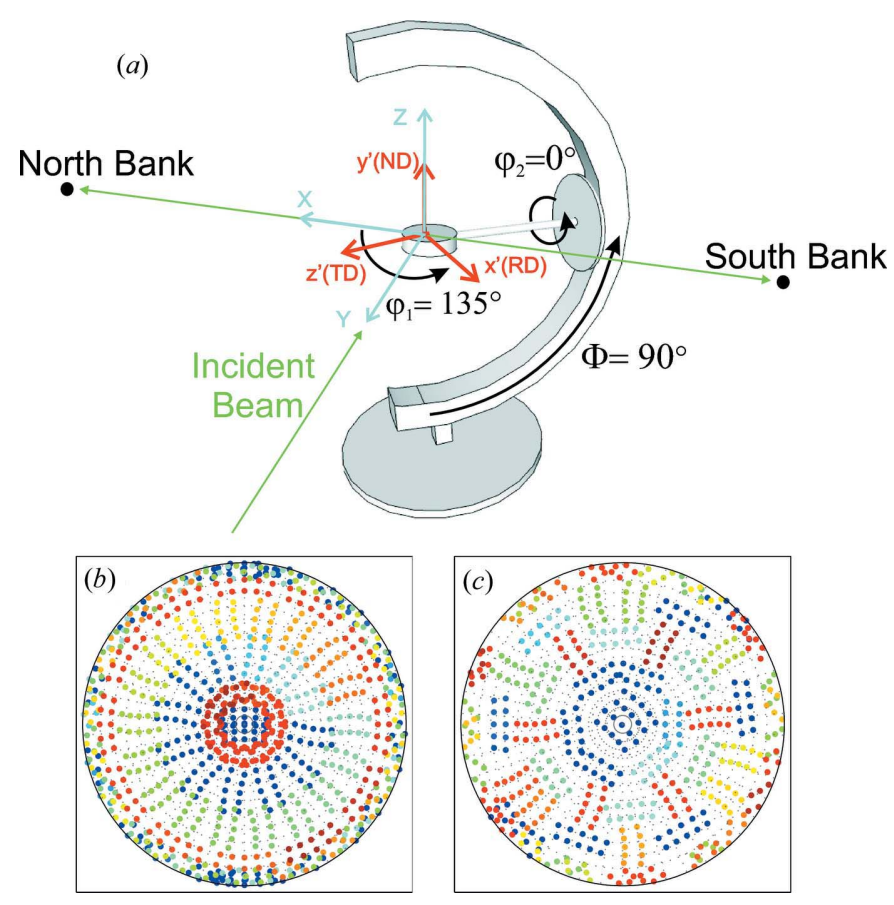

Figure 9

(a) Euler goniometer with the convention adopted for the sample $X Y Z$ (blue) and laboratory $x^{\prime} y^{\prime} z^{\prime}$ reference systems (red). (b) ENGIN-X pole figure coverage with $\sim 8 \times 8^{\circ}$ resolution using a $2 \times 5$ gridding scheme and the 45 orientations listed in Table 4. (c) Approximate equal-area grid using the 25 orientations listed in Table 5 . 
Table 5

Euler angles used for the pole figure coverage shown in Fig. 9(c).

\begin{tabular}{rrr}
\hline$\varphi_{1}\left({ }^{\circ}\right)$ & $\Phi\left({ }^{\circ}\right)$ & $\varphi_{2}\left(^{\circ}\right)$ \\
\hline 35 & 90 & 45 \\
45 & 25 & 25 \\
45 & 25 & 75 \\
45 & 25 & 95 \\
45 & 25 & 145 \\
45 & 25 & 195 \\
45 & 25 & 215 \\
45 & 25 & 265 \\
45 & 25 & 315 \\
45 & 25 & 330 \\
45 & 45 & 55 \\
45 & 45 & 110 \\
45 & 45 & 175 \\
45 & 45 & 235 \\
45 & 45 & 295 \\
45 & 45 & 355 \\
49 & 90 & 45 \\
160 & 90 & 20 \\
160 & 90 & 90 \\
160 & 90 & 140 \\
160 & 90 & 210 \\
160 & 90 & 260 \\
160 & 90 & 330 \\
180 & 90 & 00 \\
180 & 90 & 120 \\
180 & 90 & \\
\hline
\end{tabular}

complete pole figure coverage with slightly lower resolution $\left(\sim 8 \times 8^{\circ}\right)$ can be attained in a $2 \times 5$ gridding scheme of the detection banks by performing 45 specimen orientations using the Euler goniometer schematically shown in Fig. 9(a), obtaining the coverage shown in Fig. 9(b). The actual Euler angles required for such coverage are listed in Table 4. At none of the proposed angles are the incident or diffracted beams blocked by the goniometer arm. The convention adopted for the sample and laboratory reference systems is detailed in Fig. $9(a)$. The (blue) $X Y Z$ coordinate system is attached to the sample, with the $X$ axis pointing to the centre of the north detection bank, the $Y$ axis pointing in the incident beam direction and the $Z$ axis along the vertical. The (red) $x^{\prime} y^{\prime} z^{\prime}$ coordinate system is attached to the sample, which for a plate would correspond to the rolling (RD), transverse (TD) and normal (ND) directions, respectively. In the laboratory reference system, each sample orientation is defined by the goniometer Euler angles: $\varphi_{1}$ allows the whole goniometer to rotate around the $(Z)$ vertical axis; $\Phi$ allows the sample to roll over the inner semi-circle ( $X^{\prime}$ direction) and $\varphi_{2}$ represents the rotation axis around the sample holder ( $Z^{\prime}$ direction). The sample and laboratory systems coincide for $\varphi_{1}=0^{\circ}, \Phi=0^{\circ}, \varphi_{2}=$ $0^{\circ}$. In such orientation, the rolling direction points along $X$, the transverse direction along $Y$ and the normal direction along $Z$. The sample orientation in Fig. 9(a) corresponds to a $135^{\circ}$ rotation around the vertical axis, followed by a $90^{\circ}$ rotation around the $X^{\prime}$ direction, i.e. $\varphi_{1}=135^{\circ}, \Phi=90^{\circ}, \varphi_{2}=0^{\circ}$.

An alternative set of just 25 orientations, shown in Fig. 9(c) and listed in Table 5, can be used in cases where experimental time is at a premium. Such a grid gives a coarse and approximate equal-area $\mathrm{SO}(2)$ grid, following the ideas proposed by Matthies \& Wenk (1992).
The authors would like to thank Gilberto Artioli, Lyndon Edwards and Pablo Vizcaino for kindly providing some of the specimens investigated in this work. This research was partially funded by CONICET under PIP-542-2011, by IAEA under research contract $16024 / \mathrm{R} 0$ and by Universidad Nacional de Cuyo under project 06/C392.

\section{References}

Allen, A., Hutchings, M., Windsor, C. \& Andreani, C. (1985). Adv. Phys. 34, 445-473.

Artioli, G. (2007). Appl. Phys. A, 89, 899-908.

Banchik, A. D. (2009). Technical Report CNEA DD-ATN40MF-001. Comisión Nacional de Energía Atómica, Argentina.

Bouchard, P., George, D., Santisteban, J., Bruno, G., Dutta, M., Edwards, L., Kingston, E. \& Smith, D. (2005). Int. J. Press. Vessels Pip. 82, 299-310.

Bourke, M., Dunand, D. \& Ustundag, E. (2002). Appl. Phys. A, 74, s1707-s1709.

Brokmeier, H., Gan, W., Randau, C., Völler, M., Rebelo-Kornmeier, J. \& Hofmann, M. (2011). Nucl. Instrum. Methods Phys. Res. Sect. $A, 642,87-92$.

Brule, A. \& Kirstein, O. (2006). Physica B, 385-386, 1040-1042.

Dutkiewicz, J. \& Bonarski, J. (1997). Mater. Des. 18, 247-252.

Engler, O. \& Hirsch, J. (2002). Mater. Sci. Eng. A, 336, 249-262.

Fermi, E. \& Marshall, L. (1947). Phys. Rev. 71, 666-677.

Fitzpatrick, M. E. \& Lodini, A. (2003). Analysis of Residual Stress by Diffraction Using Neutron and Synchrotron Radiation London, New York: CRC Press.

Ganguly, S. (2004). PhD thesis, Open University, Milton Keynes, UK. Ganguly, S., Stelmukh, V., Edwards, L. \& Fitzpatrick, M. (2008). Mater. Sci. Eng. A, 491, 248-257.

Hannon, A. C. (2005). Nucl. Instrum. Methods Phys. Res. Sect. A, 551, 88-107.

Hielscher, R. \& Schaeben, H. (2008). J. Appl. Cryst. 41, 1024-1037.

Hirsch, J. \& Lücke, K. (1988). Acta Metall. 36, 2863-2882.

Holt, R. (2008). J. Nucl. Mater. 372, 182-214.

Hutchings, M. T., Withers, P. J., Holden, T. M. \& Lorentzen, T. (2004). Introduction to the Characterization of Residual Stress by Neutron Diffraction. Boca Raton: CRC Press.

James, J., Santisteban, J., Edwards, L. \& Daymond, M. (2004). Physica $B$, 350, e 743-e746.

Kallend, J. S., Kocks, U. F., Rollett, A. D. \& Wenk, H.-R. (1991a). Mater. Sci. Eng. A, 132, 1-11.

Kallend, J. S., Kocks, U. F., Rollett, A. D. \& Wenk, H.-R. (1991b). Textures Microstruct. 14, 1203-1208.

Kearns, J. J. (1965). Report WAPD-TM-472. Bettis Atomic Power Laboratory, West Mifflin, PA, USA.

Kockelmann, W., Chapon, L. C., Engels, R., Schelten, J., Neelmeijer, C., Walcha, H.-M., Artioli, G., Shalev, S., Perelli-Cippo, E., Tardocchi, M., Gorini, G. \& Radaelli, P. G. (2006). J. Neutron Res. 14, 37-42.

Kockelmann, W., Chapon, L. C. \& Radaelli, P. G. (2006). Physica B, 385-386, 639-643.

Kocks, U. F. (2000). Texture and Anisotropy: Preferred Orientations in Polycrystals and Their Effect on Materials Properties. Cambridge University Press.

Kong, X., Liu, W., Li, J. \& Yuan, H. (2010). J. Alloys Compd. 491, 301-307. Kropff, F., Granada, J. \& Mayer, R. (1982). Nucl. Instrum. Methods Phys. Res. 198, 515-521.

Langh, R. van, Bartoli, L., Santisteban, J. \& Visser, D. (2011). J. Anal. At. Spectrom. 26, 892-898.

Lutterotti, L., Matthies, S., Wenk, H., Schultz, A. S. \& Richardson, J. W. (1997). J. Appl. Phys. 81, 594-600.

Marshall, W. \& Lovesey, S. W. (1971). Theory of Thermal Neutron Scattering: the Use of Neutrons for the Investigation of Condensed Matter. Oxford University Press. 
Matthies, S. (2002). Mater. Sci. Forum, 408-412, 95-100.

Matthies, S., Pehl, J., Wenk, H.-R., Lutterotti, L. \& Vogel, S. C. (2005). J. Appl. Cryst. 38, 462-475.

Matthies, S. \& Wenk, H. R. (1992). Phys. Status Solidi A, 133, $253-$ 257.

Moras, K., Fischer, A. H., Klein, H. \& Bunge, H. J. (2000). J. Appl. Cryst. 33, 1162-1174.

Ray, R. K., Jonas, J. J. \& Hook, R. E. (1993). Int. Mater. Rev. 39, 129172.

Santisteban, J. R. (2005). J. Appl. Cryst. 38, 934-944.

Santisteban, J. R., Daymond, M. R., James, J. A. \& Edwards, L. (2006). J. Appl. Cryst. 39, 812-825.

Santisteban, J., Edwards, L., Fitzpatrick, M., Steuwer, A., Withers, P., Daymond, M., Johnson, M., Rhodes, N. \& Schooneveld, E. (2002). Nucl. Instrum. Methods Phys. Res. Sect. A, 481, 765-768.

Santisteban, J. R., Malamud, F., Vizcaino, P., Li, M. J., Vogel, S., Liaw, P. \& Sumin, V. I. (2014). IAEA Technical Document. In the press. International Atomic Energy Agency, Vienna, Austria.

Santisteban, J., Vicente-Alvarez, M., Vizcaino, P., Banchik, A., Vogel, S., Tremsin, A., Vallerga, J., McPhate, J., Lehmann, E. \& Kockelmann, W. (2012). J. Nucl. Mater. 425, 218-227.

Santisteban, J. R., Vizcaíno, P., Vicente-Alvarez, M. A., Malamud, F. \& Tartaglione, A. (2014). IAEA Technical Document. In the press. International Atomic Energy Agency, Vienna, Austria.

Schoenfeld, S. \& Asaro, R. (1996). Int. J. Mech. Sci. 38, 661-683.
Siano, S., Bartoli, L., Kockelmann, W., Zoppi, M. \& Miccio, M. (2004). Physica B, 350, 123-126.

Siano, S., Bartoli, L., Santisteban, J. R., Kockelmann, W., Daymond, M. R., Miccio, M. \& De Marinis, G. (2006). Archaeometry, 48, 7796.

Stelmukh, V. A. (2003). PhD thesis, Open University, Milton Keynes, UK.

Strobl, M. (2013). ODIN Instrument Proposal, Executive Summary, http://europeanspallationsource.se/imaging.

Suzuki, H., Harjo, S., Abe, J., Xu, P., Aizawa, K. \& Akita, K. (2013). Nucl. Instrum. Methods Phys. Res. Sect. A, 715, 28-38.

Ullemeyer, K., Spalthoff, P., Heinitz, J., Isakov, N., Nikitin, A. \& Weber, K. (1998). Nucl. Instrum. Methods Phys. Res. Sect. A, 412, $80-88$.

Vasudévan, A. K., Fricke, W. G., Malcolm, R. C., Bucci, R. J., Przystupa, M. A. \& Barlat, F. (1988). Metall. Trans. A, 19, 731732.

Vicente Alvarez, M., Santisteban, J., Domizzi, G. \& Almer, J. (2011). Acta Mater. 59, 2210-2220.

Walther, K., Scheffzük, C. \& Frischbutter, A. (2000). Physica B, 276278, 130-131.

Wang, X.-L., Holden, T. M., Rennich, G. Q., Stoica, A. D., Liaw, P. K., Choo, H. \& Hubbard, C. R. (2006). Physica B, 385-386, 673-675.

Wenk, H., Lutterotti, L. \& Vogel, S. (2003). Nucl. Instrum. Methods Phys. Res. Sect. A, 515, 575-588. 